\title{
Cosmology in Tension
}

5th International E-Conference on Entropy and Its Applications

\author{
Eleonora Di Valentino \\ University of Manchester
}




\section{Introduction to $\mathrm{CMB}$}
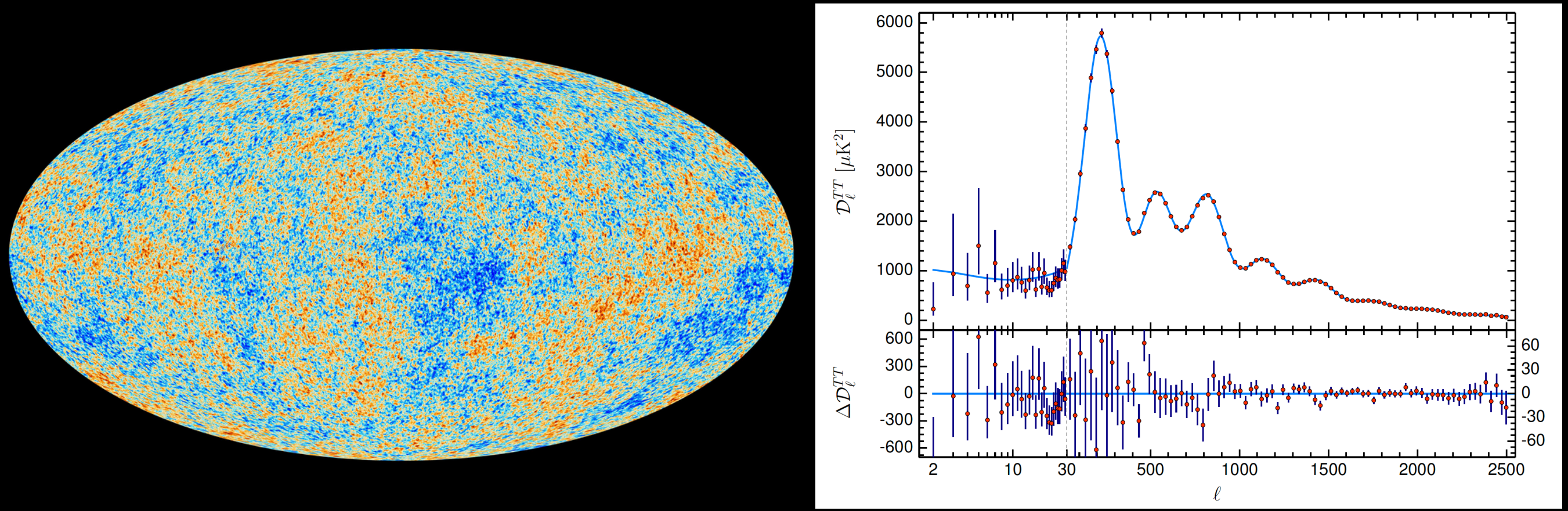

Planck collaboration, 2018

An important tool of research in cosmology is the angular power spectrum of CMB temperature anisotropies.

$$
\left\langle\frac{\Delta T}{T}\left(\overrightarrow{\gamma_{1}}\right) \frac{\Delta T}{T}\left(\overrightarrow{\gamma_{2}}\right)\right\rangle=\frac{1}{2 \pi} \sum_{\ell}(2 \ell+1) C_{\ell} P_{\ell}\left(\overrightarrow{\gamma_{1}} \cdot \overrightarrow{\gamma_{2}}\right)
$$




\section{Introduction to CMB}

Cosmological parameters: $\left(\Omega_{b} h^{2}, \Omega_{m} h^{2}, h, n_{s}, \tau, \Sigma m_{\nu}\right)$
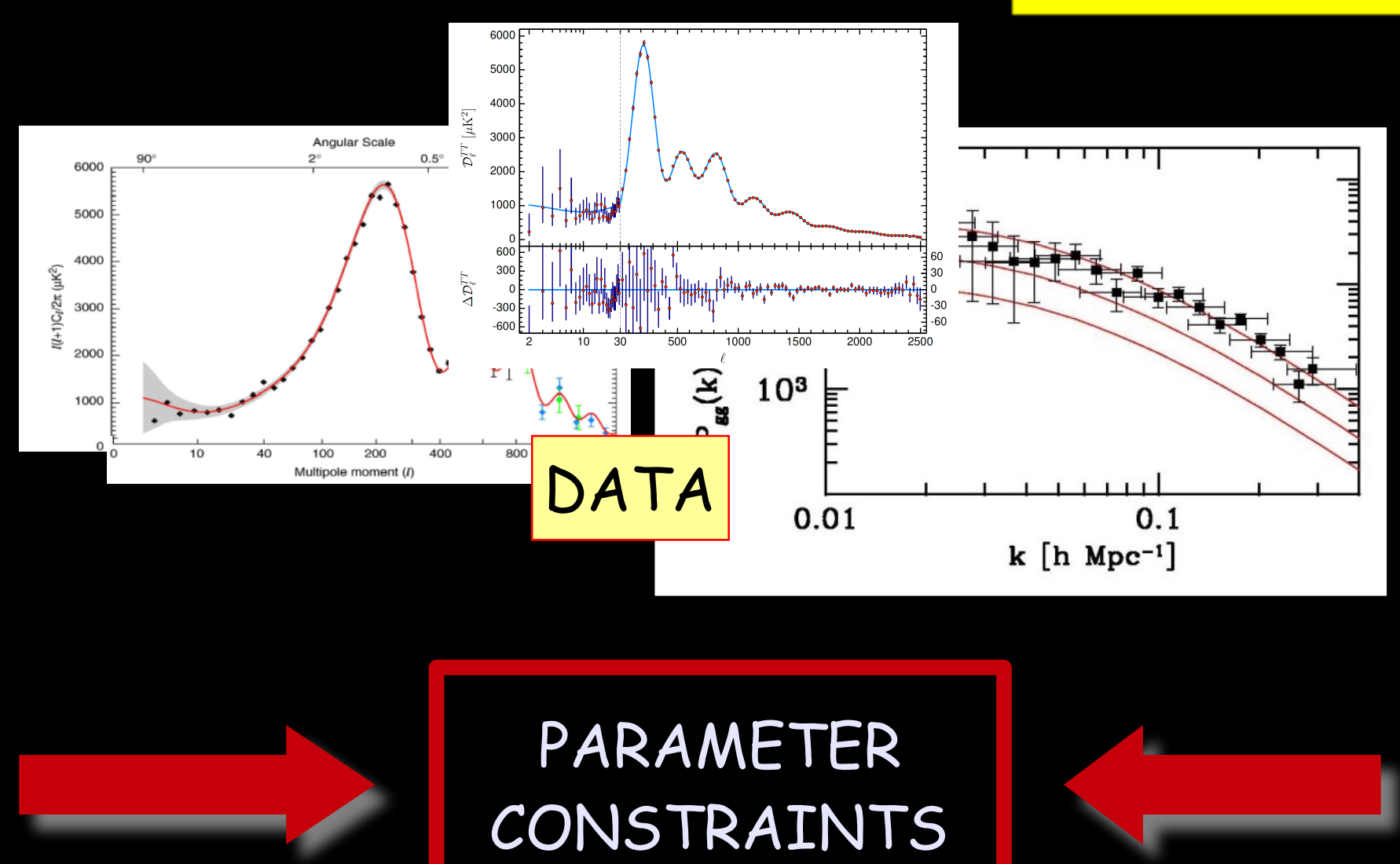

Theoretical model

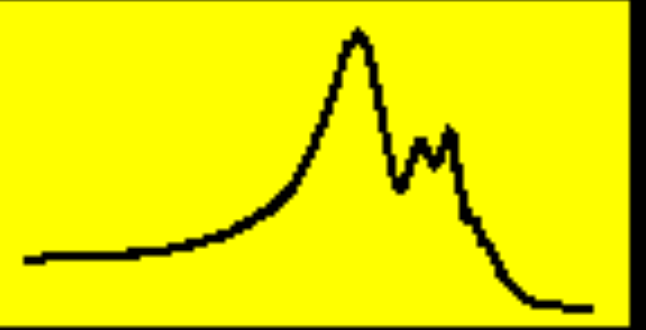

k $\left[\mathrm{h} \mathrm{Mpc}^{-1}\right]$

\section{CONSTRAINTS}




\section{Introduction to CMB}

From one side we have very accurate theoretical predictions on their angular power spectra while on the other side we have extremely precise measurements, culminated with the recent 2018 legacy release from the Planck satellite experiment. 


\section{CMB constraints}

Planck $\mathrm{EE}+$ lowE $+\mathrm{BAO}$

Planck TE+lowE

Planck TT+lowE

Planck TT, TE,EE+lowE

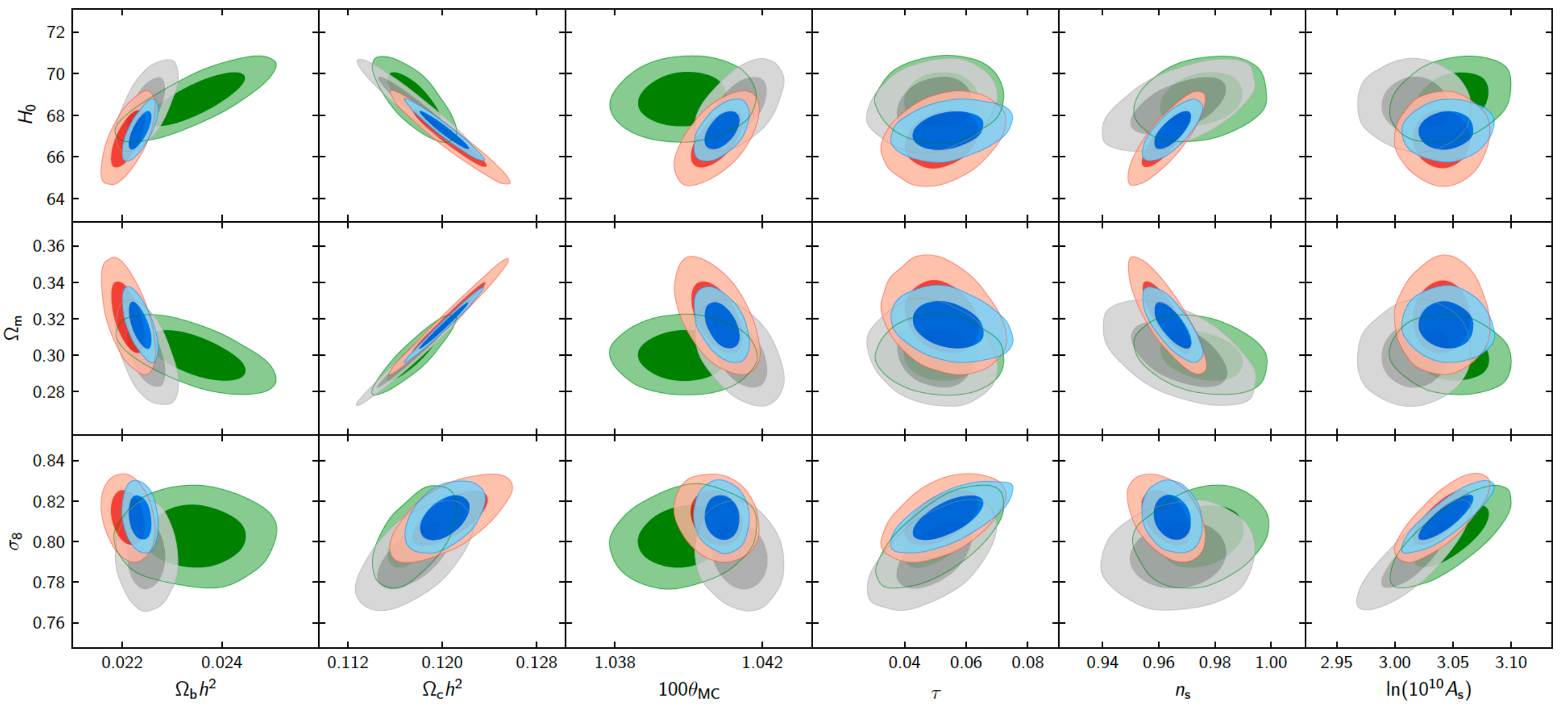

Planck 2018, Aghanim et al., arXiv:1807.06209 [astro-ph.CO]

Constraints on parameters of the base-LCDM model from the separate Planck EE, TE, and TT high-I spectra combined with low-I polarization (lowE), and, in the case of EE also with BAO, compared to the joint result using Planck TT,TE,EE+lowE. 


\section{CMB constraints}

\begin{tabular}{|c|c|c|c|c|c|c|}
\hline Parameter & $\begin{array}{l}\mathrm{TT}+\text { lowE } \\
68 \% \text { limits }\end{array}$ & $\begin{array}{l}\text { TE+lowE } \\
68 \% \text { limits }\end{array}$ & $\begin{array}{l}\text { EE+lowE } \\
68 \% \text { limits }\end{array}$ & $\begin{array}{c}\text { TT,TE,EE+lowE } \\
68 \% \text { limits } \\
\end{array}$ & $\begin{array}{c}\text { TT,TE,EE+lowE+lensing } \\
68 \% \text { limits } \\
\end{array}$ & $\begin{array}{c}\text { TT,TE,EE+lowE+lensing }+ \text { BAO } \\
68 \% \text { limits }\end{array}$ \\
\hline$\Omega_{\mathrm{b}} h^{2} \ldots \ldots \ldots$ & $0.02212 \pm 0.00022$ & $0.02249 \pm 0.00025$ & $0.0240 \pm 0.0012$ & $0.02236 \pm 0.00015$ & $0.02237 \pm 0.00015$ & $0.02242 \pm 0.00014$ \\
\hline$\Omega_{\mathrm{c}} h^{2} \ldots \ldots \ldots$ & $0.1206 \pm 0.0021$ & $0.1177 \pm 0.0020$ & $0.1158 \pm 0.0046$ & $0.1202 \pm 0.0014$ & $0.1200 \pm 0.0012$ & $0.11933 \pm 0.00091$ \\
\hline $100 \theta_{\mathrm{MC}} \ldots \ldots$ & $1.04077 \pm 0.00047$ & $1.04139 \pm 0.00049$ & $1.03999 \pm 0.00089$ & $1.04090 \pm 0.00031$ & $1.04092 \pm 0.00031$ & $1.04101 \pm 0.00029$ \\
\hline$\tau \ldots \ldots$ & $0.0522 \pm 0.0080$ & $0.0496 \pm 0.0085$ & $0.0527 \pm 0.0090$ & $0.0544_{-0.0081}^{+0.0070}$ & $0.0544 \pm 0.0073$ & $0.0561 \pm 0.0071$ \\
\hline $\ln \left(10^{10} A_{\mathrm{s}}\right) \ldots \ldots$ & $3.040 \pm 0.016$ & $3.018_{-0.018}^{+0.020}$ & $3.052 \pm 0.022$ & $3.045 \pm 0.016$ & $3.044 \pm 0.014$ & $3.047 \pm 0.014$ \\
\hline$n_{\mathrm{s}} \ldots \ldots \ldots$ & $0.9626 \pm 0.0057$ & $0.967 \pm 0.011$ & $0.980 \pm 0.015$ & $0.9649 \pm 0.0044$ & $0.9649 \pm 0.0042$ & $0.9665 \pm 0.0038$ \\
\hline$H_{0}\left[\mathrm{~km} \mathrm{~s}^{-1} \mathrm{Mpc}^{-1}\right] \ldots$ & $66.88 \pm 0.92$ & $68.44 \pm 0.91$ & $69.9 \pm 2.7$ & $67.27 \pm 0.60$ & $67.36 \pm 0.54$ & $67.66 \pm 0.42$ \\
\hline$\Omega_{\Lambda} \ldots \ldots \ldots$ & $0.679 \pm 0.013$ & $0.699 \pm 0.012$ & $0.711_{-0.026}^{+0.033}$ & $0.6834 \pm 0.0084$ & $0.6847 \pm 0.0073$ & $0.6889 \pm 0.0056$ \\
\hline$\Omega_{\mathrm{m}} \ldots \ldots \ldots$ & $0.321 \pm 0.013$ & $0.301 \pm 0.012$ & $0.289_{-0.033}^{+0.026}$ & $0.3166 \pm 0.0084$ & $0.3153 \pm 0.0073$ & $0.3111 \pm 0.0056$ \\
\hline$\Omega_{\mathrm{m}} h^{2} \ldots \ldots \ldots$ & $0.1434 \pm 0.0020$ & $0.1408 \pm 0.0019$ & $0.1404_{-0.0039}^{+0.0034}$ & $0.1432 \pm 0.0013$ & $0.1430 \pm 0.0011$ & $0.14240 \pm 0.00087$ \\
\hline$\Omega_{\mathrm{m}} h^{3} \ldots \ldots \ldots$ & $0.09589 \pm 0.00046$ & $0.09635 \pm 0.00051$ & $0.0981_{-0.0018}^{+0.0016}$ & $0.09633 \pm 0.00029$ & $0.09633 \pm 0.00030$ & $0.09635 \pm 0.00030$ \\
\hline$\sigma_{8} \ldots \ldots \ldots$ & $0.8118 \pm 0.0089$ & $0.793 \pm 0.011$ & $0.796 \pm 0.018$ & $0.8120 \pm 0.0073$ & $0.8111 \pm 0.0060$ & $0.8102 \pm 0.0060$ \\
\hline$S_{8} \equiv \sigma_{8}\left(\Omega_{\mathrm{m}} / 0.3\right)^{0.5}$ & $0.840 \pm 0.024$ & $0.794 \pm 0.024$ & $0.781_{-0.060}^{+0.052}$ & $0.834 \pm 0.016$ & $0.832 \pm 0.013$ & $0.825 \pm 0.011$ \\
\hline
\end{tabular}

Planck 2018, Aghanim et al., arXiv:1807.06209 [astro-ph.CO]

The precision measurements of the $\mathrm{CMB}$ polarization spectra have the potential to constrain cosmological parameters to higher accuracy than measurements of the temperature spectra because the acoustic peaks are narrower in polarization and unresolved foreground contributions at high multipoles are much lower in polarization than in temperature.

2018 Planck results are perfectly in agreement with the standard ^CDM cosmological model. 
However, anomalies and tensions between Planck and other cosmological probes are present well above the 3 standard deviations. These discrepancies, already hinted in previous Planck data releases, have persisted and strengthened despite several years of accurate analyses.

Last year, the Royal Astronomical Society awarded Planck their Group Achievement Award with the citation "(Planck) has now ushered in an era of tension cosmology.", clearly indicating that these tensions have reached such a level of statistical significance that the understanding of their physical nature is of utmost importance for modern cosmology.

If not due to systematics, the current anomalies could represent a crisis for the standard cosmological model and their experimental confirmation can bring a revolution in our current ideas of the structure and evolution of the Universe. 
The most famous and persisting anomalies and tensions of the CMB are:

- H0 with local measurements

- S8 with cosmic shear data

- AL internal anomaly

- $\Omega K$ different from zero 


\section{The $\mathrm{HO}$ tension at more than $3 \sigma$}

CMB: in this case the cosmological constraints are obtained by assuming a cosmological model and are therefore model dependent. Moreover these bounds are also affected by the degeneracy between the parameters that induce similar effects on the observables. Therefore the Planck constraints can change when modifying the assumptions of the underlying cosmological model.

$$
\mathrm{HO}=67.27 \pm 0.60 \mathrm{~km} / \mathrm{s} / \mathrm{Mpc} \text { in } \Lambda \mathrm{CDM}
$$

Planck 2018, Aghanim et al., arXiv:1807.06209 [astro-ph.CO]

Direct local distance ladder measurements: the 2016 estimate of the Hubble constant is based on Supernovae type-la measurements, obtained combining three different geometric distance calibrations of Cepheids,

$$
\begin{gathered}
\mathrm{HO}=73.24 \pm 1.74 \mathrm{~km} / \mathrm{s} / \mathrm{Mpc} \\
\text { Riess et al. Astrophys.J. 826, no. 1, } 56 \text { (2016) }
\end{gathered}
$$

The 2018 estimate include parallax measurements of 7 long-period ( $>10$ days) Milky Way Cepheids using astrometry from spatial scanning of WFC3 on HST. 


\section{The $\mathrm{HO}$ tension at more than $4 \sigma$}

Table 5. Best Estimates of $\mathrm{H}_{0}$ Including Systematics

\begin{tabular}{|c|c|c|}
\hline Anchor(s) & $\begin{array}{c}\text { Value } \\
{\left[\mathrm{km} \mathrm{s}^{-1} \mathrm{Mpc}^{-1}\right]}\end{array}$ & $\begin{array}{l}\Delta \text { Planck }^{*}+ \\
\Lambda \mathrm{CDM}(\sigma)\end{array}$ \\
\hline LMC & $74.22 \pm 1.82$ & 3.6 \\
\hline \multicolumn{3}{|l|}{ Two anchors } \\
\hline LMC + NGC 4258 & $73.40 \pm 1.55$ & 3.7 \\
\hline $\mathrm{LMC}+\mathrm{MW}$ & $74.47 \pm 1.45$ & 4.6 \\
\hline NGC $4258+\mathrm{MW}$ & $73.94 \pm 1.58$ & 3.9 \\
\hline \multicolumn{3}{|l|}{ Three anchors (preferred) } \\
\hline NGC $4258+\mathrm{MW}+\mathrm{LMC}$ & $74.03 \pm 1.42$ & 4.4 \\
\hline
\end{tabular}

Riess et al. arXiv:1903.07603 [astro-ph.CO]

Recently, the $\mathrm{HO}$ measurement has been improved using Hubble Space Telescope observations of 70 long-period Cepheids in the Large Magellanic Cloud.

The tension becomes of $4.4 \sigma$ between the local measurement of $\mathrm{HO}$ and $_{10}$ the value predicted from Planck in $\Lambda$ CDM. 


\section{The $\mathrm{HO}$ tension at more than $5 \sigma$}

$\mathrm{CMB}: \quad \mathrm{HO}=67.27 \pm 0.60 \mathrm{~km} / \mathrm{s} / \mathrm{Mpc}$ in $\Lambda \mathrm{CDM}$ $\mathrm{BAO}+$ Pantheon $+\mathrm{BBN}+\theta_{\mathrm{MC}}$, Planck: $\mathrm{HO}=67.9 \pm 0.8 \mathrm{~km} / \mathrm{s} / \mathrm{Mpc}$ Planck 2018, Aghanim et al., arXiv:1807.06209 [astro-ph.CO]

SHOES: $\quad H O=74.03 \pm 1.42 \mathrm{~km} / \mathrm{s} / \mathrm{Mpc}$ Riess et al. arXiv:1903.07603 [astro-ph.CO]
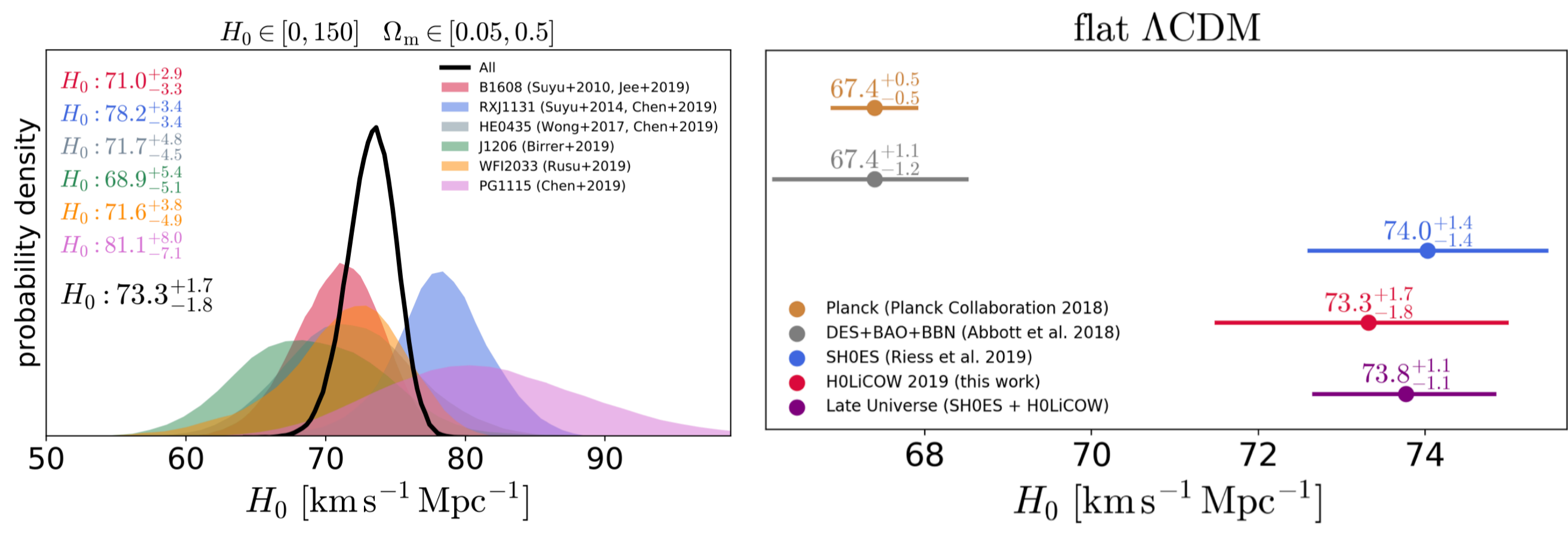

Strong Lensing: Multiply-imaged quasar systems through strong gravitational lensing made by the HoliCOW collaboration $\mathrm{HO}=73.3+1.7-1.8 \mathrm{~km} / \mathrm{s} / \mathrm{Mpc}$

Wong et al. arXiv:1907.04869v1 
Since the Planck constraints are model dependent, we can try to expand the cosmological scenario and see which extensions work in solving the tensions between the cosmological probes.

For example, the most famous extensions for solving the $\mathrm{HO}$ tension are:

\section{the neutrino effective number the dark energy equation of state}




\section{The Neutrino effective number}

If we compare the Planck 2015 constraint on Neff at $68 \% \mathrm{cl}$

$$
\begin{array}{ll}
N_{\mathrm{eff}}=3.13 \pm 0.32 & \text { Planck } \mathrm{TT}+\text { low } \mathrm{P} \\
N_{\mathrm{eff}}=3.15 \pm 0.23 & \text { Planck } \mathrm{TT}+\text { low }+\mathrm{BAO}
\end{array}
$$

with the new Planck 2018 bound,

$N_{\mathrm{eff}}=2.92_{-0.37}^{+0.36} \quad(95 \%$, Planck TT,TE,EE+lowE $)$,

we see that the neutrino effective number is now very well constrained.

The main reason for this good accuracy is due to the lack of the early integrated Sachs Wolfe effect in polarization data. The inclusion of polarization helps in determining the amplitude of the eISW and Neff. HO passes from $68.0 \pm 2.8 \mathrm{~km} / \mathrm{s} / \mathrm{Mpc}$ (2015) to $66.4 \pm 1.4 \mathrm{~km} / \mathrm{s} / \mathrm{Mpc}$ (2018), and the tension with Riess+19 increases from 2.10 to $3.8 \sigma$ also varying Neff.

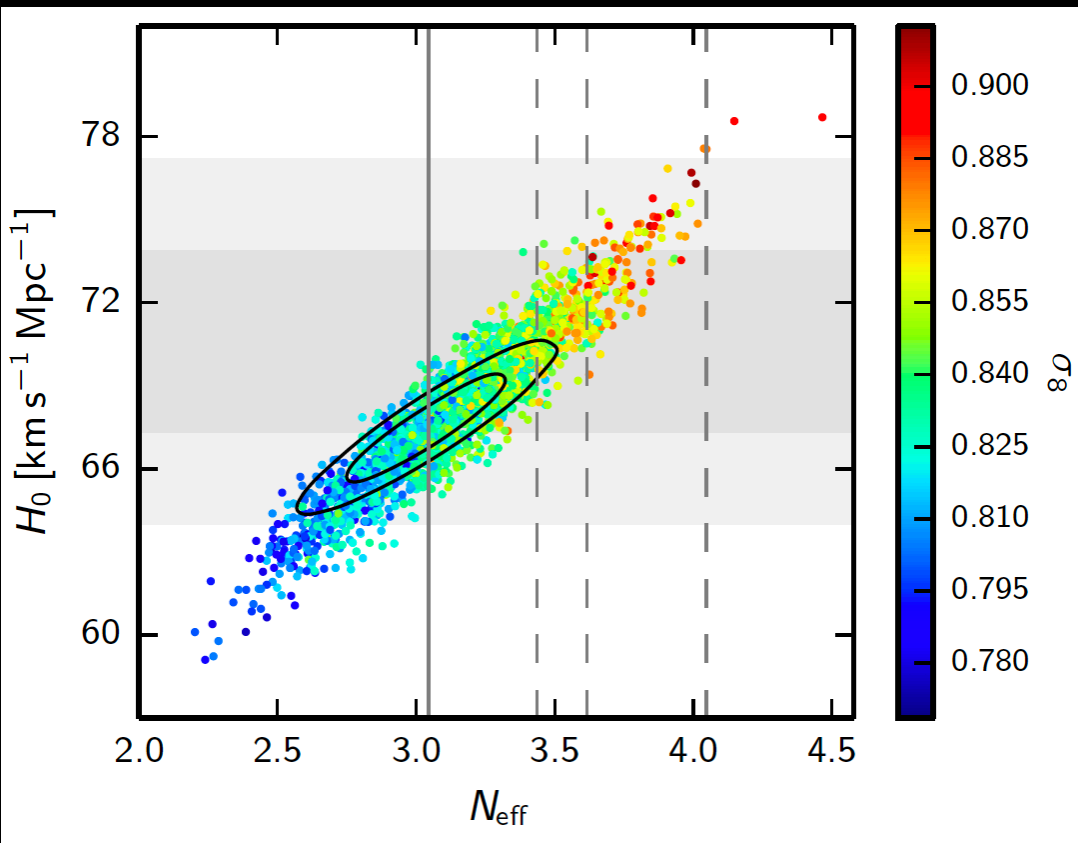

Planck collaboration, 2015

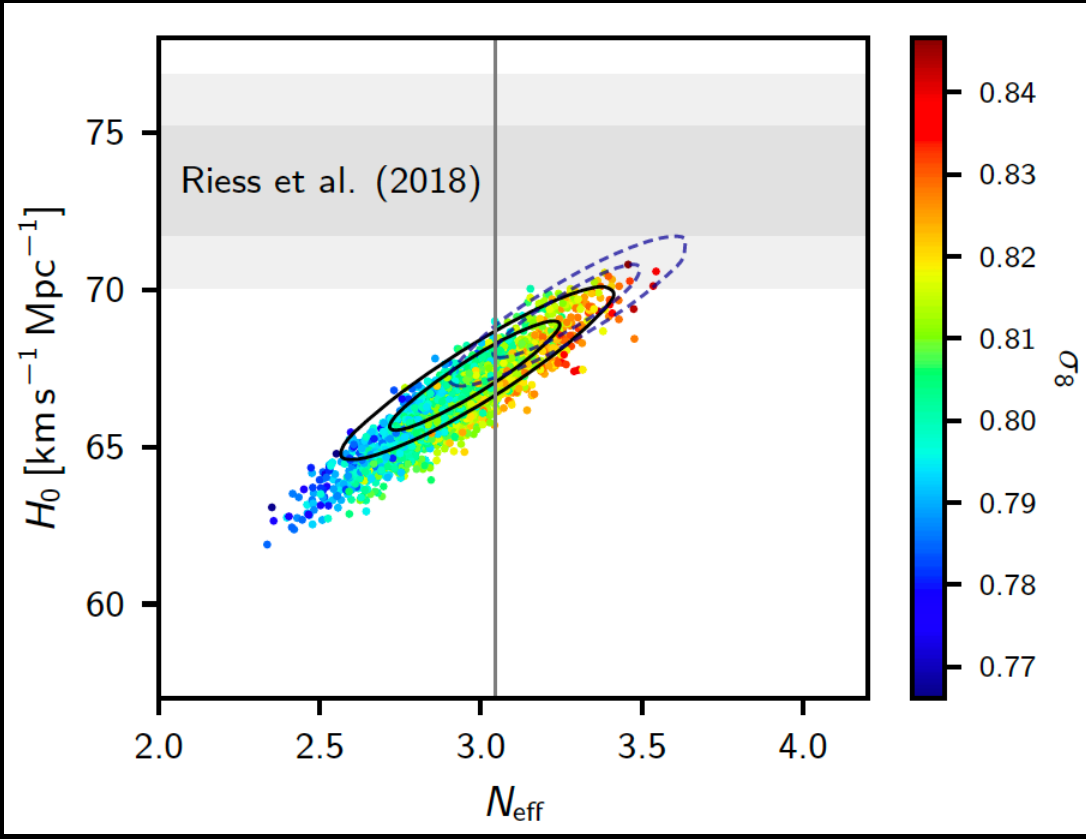

Planck collaboration, 2018 


\section{The Dark energy equation of state}

Changing the dark energy equation of state $\mathrm{w}$, we are changing the expansion rate of the Universe:

$$
\begin{aligned}
H^{2}=H_{0}^{2} & {\left[\Omega_{m}(1+z)^{3}+\Omega_{r}(1+z)^{4}\right.} \\
& \left.+\Omega_{\mathrm{de}}(1+z)^{3(1+w)}+\Omega_{k}(1+z)^{2}\right]
\end{aligned}
$$

w introduces a geometrical degeneracy with the Hubble constant that will be unconstrained using the CMB data only, resulting in agreement with Riess+19.

We have in $2018 \mathrm{w}=-1.58^{+0.52}-0.41$ with $\mathrm{HO}>69.9 \mathrm{~km} / \mathrm{s} / \mathrm{Mpc}$ at $95 \%$ c.l.

Planck data prefer a phantom dark energy, with an energy component with $\mathrm{w}<$ -1 , for which the density increases with time in an expanding universe that will end in a Big Rip. A phantom dark energy violates the energy condition $\rho \geq \mid \mathrm{l}$, that means that the matter could move faster than light and a comoving observer measure a negative energy density, and the Hamiltonian could have vacuum instabilities due to a negative kinetic energy.

Anyway, there exist models that expect an effective energy density with a phantom equation of state without showing the problems before, as for example the Parker Vacuum Metamorphosis Di Valentino et al., Phys.Rev. D97 (2018) no.4, 043528. 


\section{Less famous extensions for solving the $\mathrm{HO}$ tension are:}

- Interacting dark sector (Di Valentino et al. arXiv:1704.08342, Kumar and Nunes arXiv:1702.02143, Yang et al. arXiv:1805.08252, Yang et al. arXiv:1809.06883, Yang et al. arXiv:1906.11697, Martinelli et al. arXiv:1902.10694, Di Valentino et al. 2019, etc...)

- Parker Vacuum Metamorphosis (Di Valentino et al. 2018)

- Vacuum Dynamics (Sola Peracaula et al. arXivi:1703.08218)

- Early dark Energy (Poulin et al. arXiv:1811.04083)

- Uber-gravity (Khosravi et al. arXiv:1710.09366)

- Bulk viscosity (Yang et al. arXiv:1906.04162)

- Decaying dark matter (Pandey et al. arXiv:1902.10636, Vattis et al. arXiv:1903.06220, etc..)

- Many many otherS... (Colgain et al. arXiv:1807.07451, Nunes arXiv:1802.02281, Agrawal et al. arXiv:1904.01016, Yang et al. arXiv:1907.05344, Martinelli and Tutusaus arXiv:1906.09189, Adhikari and Huterer arXiv:1905.02278, Gelmini et al. arXiv:1906.10136, etc..) 


\section{IDE can solve the $\mathrm{H} 0$ tension}

In the standard cosmological framework, the dark matter is assumed to be collisionless. In practice this means that one arbitrarily sets the dark matter interactions to zero when predicting the angular power spectrum of the CMB.

In particular, dark matter and dark energy are described as separate fluids not sharing interactions beyond gravitational ones. However, from a microphysical perspective it is hard to imagine how non-gravitational DM-DE interactions can be avoided, unless forbidden by a fundamental symmetry. This has motivated a large number of studies based on models where DM and DE share interactions other than gravitational. 


\section{IDE can solve the $\mathrm{H} 0$ tension}

If we consider the interacting dark energy scenario characterised by a modification to the usual conservation equations, with the introduction of an interaction:

Dark matter and Dark Energy energy-momentum tensor

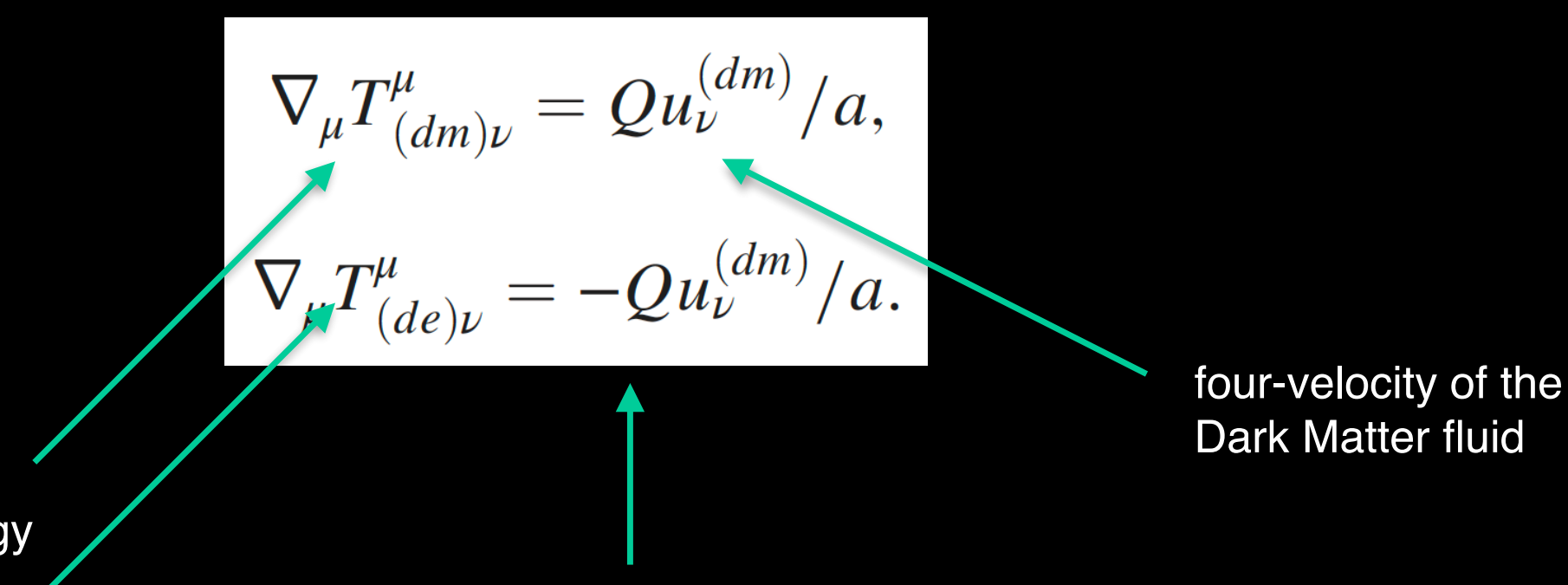

Interaction rate

With the interaction rate proportional to the dark energy density $\rho_{\mathrm{de}}$ via a negative dimensionless parameter $\xi$ quantifying the strength of the coupling, to avoid early-time instabilities. 


\section{Planck 2018}

In this scenario of IDE the tension on $\mathrm{HO}$ between the Planck satellite and R19 is completely solved. The coupling could affect the value of the present matter energy density $\Omega_{\mathrm{m}}$. Therefore, if within an interacting model $\Omega_{\mathrm{m}}$ is smaller (because for negative $\xi$ the dark matter density will decay into the dark energy one), a larger value of $\mathrm{HO}$ would be required in order to satisfy the peaks structure of CMB observations, which accurately determine the value of $\Omega_{\mathrm{m}} \mathrm{h}^{2}$.

\begin{tabular}{ccc}
\hline \hline Parameter & Planck & Planck+R19 \\
\hline$\Omega_{\mathrm{b}} h^{2}$ & $0.02239 \pm 0.00015$ & $0.02239 \pm 0.00015$ \\
$\Omega_{\mathrm{c}} h^{2}$ & $<0.105$ & $<0.0615$ \\
$n_{s}$ & $0.9655 \pm 0.0043$ & $0.9656 \pm 0.0044$ \\
$100 \theta_{\mathrm{s}}$ & $1.0458_{-0.0021}^{+0.0033}$ & $1.0470 \pm 0.0015$ \\
$\tau$ & $0.0541 \pm 0.0076$ & $0.0534 \pm 0.0080$ \\
$\xi$ & $-0.54_{-0.28}^{+0.12}$ & $-0.66_{-0.13}^{+0.09}$ \\
\hline$H_{0}\left[\mathrm{~km} \mathrm{~s}^{-1} \mathrm{Mpc}^{-1}\right]$ & $72.8_{-1.5}^{+3.0}$ & $74.0_{-1.0}^{+1.2}$ \\
\hline
\end{tabular}

TABLE I. Mean values with their $68 \%$ C.L. errors on selected cosmological parameters within the $\xi \Lambda \mathrm{CDM}$ model, considering either the Planck 2018 legacy dataset alone, or the same dataset in combination with the $R 19$ Gaussian prior on $H_{0}$ based on the latest local distance measurement from $H S T$. The quantity quoted in the case of $\Omega_{\mathrm{c}} h^{2}$ is the $95 \%$ C.L. upper limit. 


\section{Planck 2018}

Therefore we can safely combine the two datasets together, and we obtain a nonzero dark matter-dark energy coupling $\xi$ at more than FIVE standard deviations.

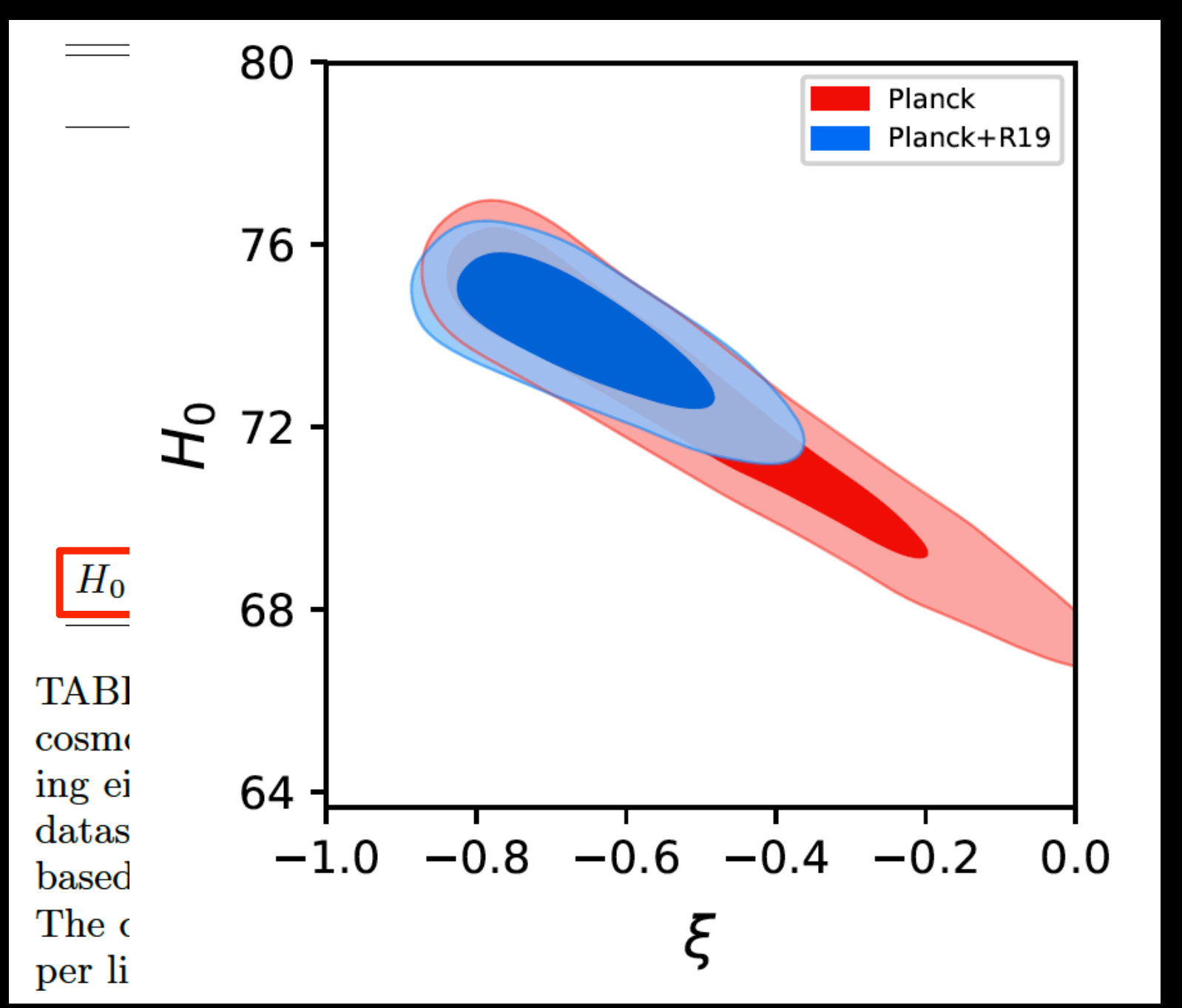

Di Valentino et al. arXiv:1908.04281 


\section{Bayes factor}

Anyway it is clearly interesting to quantify the better accordance of a model with the data respect to another by using the marginal likelihood also known as the Bayesian evidence.

Given a vector of parameters $\theta$ of a model $M$ and a set of data $x$, the parameters posterior distribution is given by

The marginal likelihood (or evidence) given by

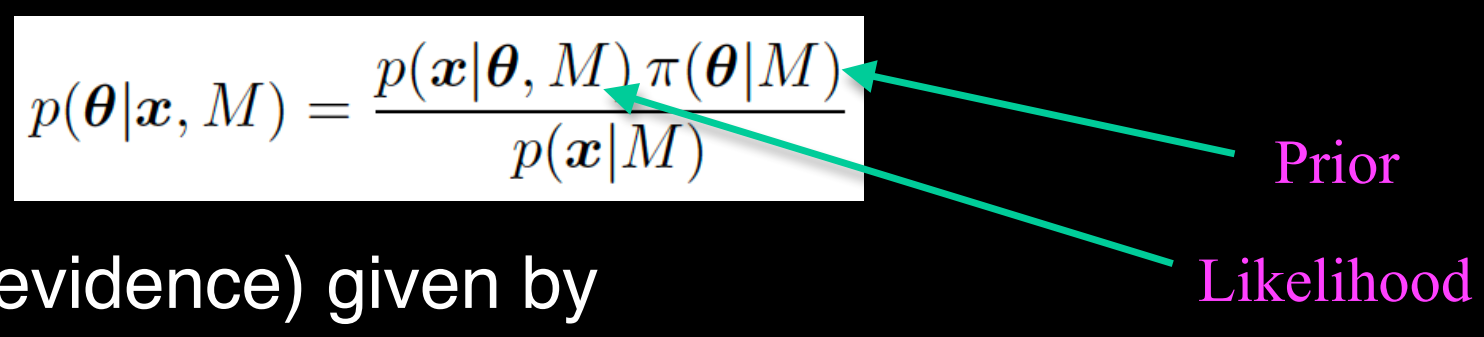

$$
E \equiv p(\boldsymbol{x} \mid M)=\int d \boldsymbol{\theta} p(\boldsymbol{x} \mid \boldsymbol{\theta}, M) \pi(\boldsymbol{\theta} \mid M)
$$

Given two competing models $\mathrm{M}_{0}$ and $\mathrm{M}_{1}$ it is useful to consider the ratio of the likelihood probability (the Bayes factor):

$$
\ln \mathcal{B}=p\left(\boldsymbol{x} \mid M_{0}\right) / p\left(\boldsymbol{x} \mid M_{1}\right)
$$

According to the revised Jeffrey's scale by Kass and Raftery 1995, the evidence for Mo (against $\mathrm{M}_{1}$ ) is considered as "positive" if I $\operatorname{lnB} \mid>1.0$, "strong" if $|\operatorname{lnB}|>3.0$, and "very strong" if I InB I > 5.0. 


\section{Planck 2018}

Computing the Bayes factor for the IDE model with respect to LCDM for the Planck dataset we find $\ln B=1.2$, ie a positive preference for the IDE model. If we consider Planck + R19 we find the extremely high value InB $=10.0$, indicating a very strong preference for the IDE model.

\begin{tabular}{ccc}
\hline \hline Parameter & Planck & Planck+R19 \\
\hline$\Omega_{\mathrm{b}} h^{2}$ & $0.02239 \pm 0.00015$ & $0.02239 \pm 0.00015$ \\
$\Omega_{\mathrm{c}} h^{2}$ & $<0.105$ & $<0.0615$ \\
$n_{s}$ & $0.9655 \pm 0.0043$ & $0.9656 \pm 0.0044$ \\
$100 \theta_{\mathrm{s}}$ & $1.0458_{-0.0021}^{+0.0033}$ & $1.0470 \pm 0.0015$ \\
$\tau$ & $0.0541 \pm 0.0076$ & $0.0534 \pm 0.0080$ \\
$\xi$ & $-0.54_{-0.28}^{+0.12}$ & $-0.66_{-0.13}^{+0.09}$ \\
$H_{0}\left[\mathrm{~km} \mathrm{~s}^{-1} \mathrm{Mpc}^{-1}\right]$ & $72.8_{-1.5}^{+3.0}$ & $74.0_{-1.0}^{+1.2}$ \\
\hline
\end{tabular}

TABLE I. Mean values with their $68 \%$ C.L. errors on selected cosmological parameters within the $\xi \Lambda$ CDM model, considering either the Planck 2018 legacy dataset alone, or the same dataset in combination with the $R 19$ Gaussian prior on $H_{0}$ based on the latest local distance measurement from $H S T$. The quantity quoted in the case of $\Omega_{\mathrm{c}} h^{2}$ is the $95 \%$ C.L. upper limit.

Di Valentino et al. arXiv:1908.04281 


\section{Planck 2018}

Di Valentino et al. arXiv:1910.09853

Parameters

Planck

Planck

Planck

Planck

Planck

$+\mathrm{R} 19$

+lensing

$+\mathrm{BAO}$

+ Pantheon

\begin{tabular}{cccccc}
$\Omega_{b} h^{2}$ & $0.02239 \pm 0.00015$ & $0.02239 \pm 0.00015$ & $0.02241 \pm 0.00014$ & $0.02236 \pm 0.00014$ & $0.02235 \pm 0.00015$ \\
$\Omega_{c} h^{2}$ & $<0.0634$ & $0.031_{-0.023}^{+0.013}$ & $<0.0675$ & $0.095_{-0.008}^{+0.022}$ & $0.103_{-0.007}^{+0.013}$ \\
$100 \theta_{\mathrm{MC}}$ & $1.0458_{-0.0021}^{+0.0033}$ & $1.0470 \pm 0.0015$ & $1.0456_{-0.0024}^{+0.0031}$ & $1.0424_{-0.0013}^{+0.0006}$ & $1.04185_{-0.00078}^{+0.00049}$ \\
$\tau$ & $0.0541 \pm 0.0076$ & $0.0534 \pm 0.0080$ & $0.0526 \pm 0.0074$ & $0.0540 \pm 0.0076$ & $0.0540 \pm 0.0076$ \\
$n_{s}$ & $0.9655 \pm 0.0043$ & $0.9656 \pm 0.0044$ & $0.9663 \pm 0.0040$ & $0.9647 \pm 0.0040$ & $0.9643 \pm 0.0042$ \\
$\ln \left(10^{10} A_{s}\right)$ & $3.044 \pm 0.016$ & $3.042 \pm 0.017$ & $3.039_{-0.015}^{+0.013}$ & $3.044 \pm 0.016$ & $3.044 \pm 0.016$ \\
$\xi$ & $-0.54_{-0.28}^{+0.12}$ & $-0.66_{-0.13}^{+0.09}$ & $-0.51_{-0.29}^{+0.12}$ & $-0.22_{-0.05}^{+0.21}$ & $-0.15_{-0.06}^{+0.12}$ \\
$H_{0}[\mathrm{~km} / \mathrm{s} / \mathrm{Mpc}]$ & $72.8_{+1.5}^{+3.0}$ & $74.0_{-1.0}^{+1.2}$ & $72.8_{+1.6}^{+3.0}$ & $69.4_{-1.5}^{+0.9}$ & $68.6_{-1.0}^{+0.8}$ \\
$\sigma_{8}$ & $2.3_{-1.4}^{+0.4}$ & $2.71_{-1.3}^{+0.05}$ & $2.2_{-1.4}^{+0.4}$ & $1.05_{-0.24}^{+0.03}$ & $0.95_{-0.12}^{+0.04}$ \\
$S_{8}$ & $1.30_{-0.44}^{+0.17}$ & $1.44_{-0.34}^{+0.17}$ & $1.30_{-0.42}^{+0.15}$ & $0.93_{-0.10}^{+0.03}$ & $0.892_{-0.054}^{+0.028}$ \\
\hline \hline
\end{tabular}

The addition of low-redshift measurements, as BAO or Pantheon data, still hints to the presence of a coupling, albeit at a lower statistical significance.

Also for these two data sets the Hubble constant values are larger than those obtained in the case of a pure LCDM scenario, enough to bring the $\mathrm{HO}$ tension well below the $3 \sigma$ from $4.4 \sigma$. 


\section{Planck 2018}

In other words, the tension between Planck+BAO and R19 could be due to a statistical fluctuation in this case.

Moreover, BAO data is extracted under the assumption of LCDM, and the modified scenario of interacting dark energy could affect the result. In fact, the full procedure which leads to the BAO constraints carried out by the different collaborations might be not necessarily valid in extended DE models.

For instance, the BOSS collaboration advises caution when using their BAO measurements (both the pre- and post reconstruction measurements) in more exotic dark energy cosmologies.

BAO constraints themselves might need to be revised in a non-trivial manner when applied to constrain extended dark energy cosmologies. 


\section{$A_{L}$ internal anomaly}

CMB photons emitted at recombination are deflected by the gravitational lensing effect of massive cosmic structures.

The lensing amplitude AL parameterizes the rescaling of the lensing potential $\phi(n)$, then the power spectrum of the lensing field:

$$
C_{\ell}^{\phi \phi} \rightarrow A_{\mathrm{L}} C_{\ell}^{\phi \phi}
$$

The gravitational lensing deflects the photon path

by a quantity defined by the gradient of the lensing potential $\phi(n)$, integrated along the line of sight $n$, remapping the temperature field. 


\section{$A_{L}$ internal anomaly}

Its effect on the power spectrum is the smoothing of the acoustic peaks, increasing AL.

Interesting consistency checks is if the amplitude of the smoothing effect in the

CMB power spectra matches the theoretical expectation $\mathrm{AL}=1$ and whether the amplitude of the smoothing is consistent with that measured by the lensing reconstruction.

If $A L=1$ then the theory is correct, otherwise we have a new physics or systematics.

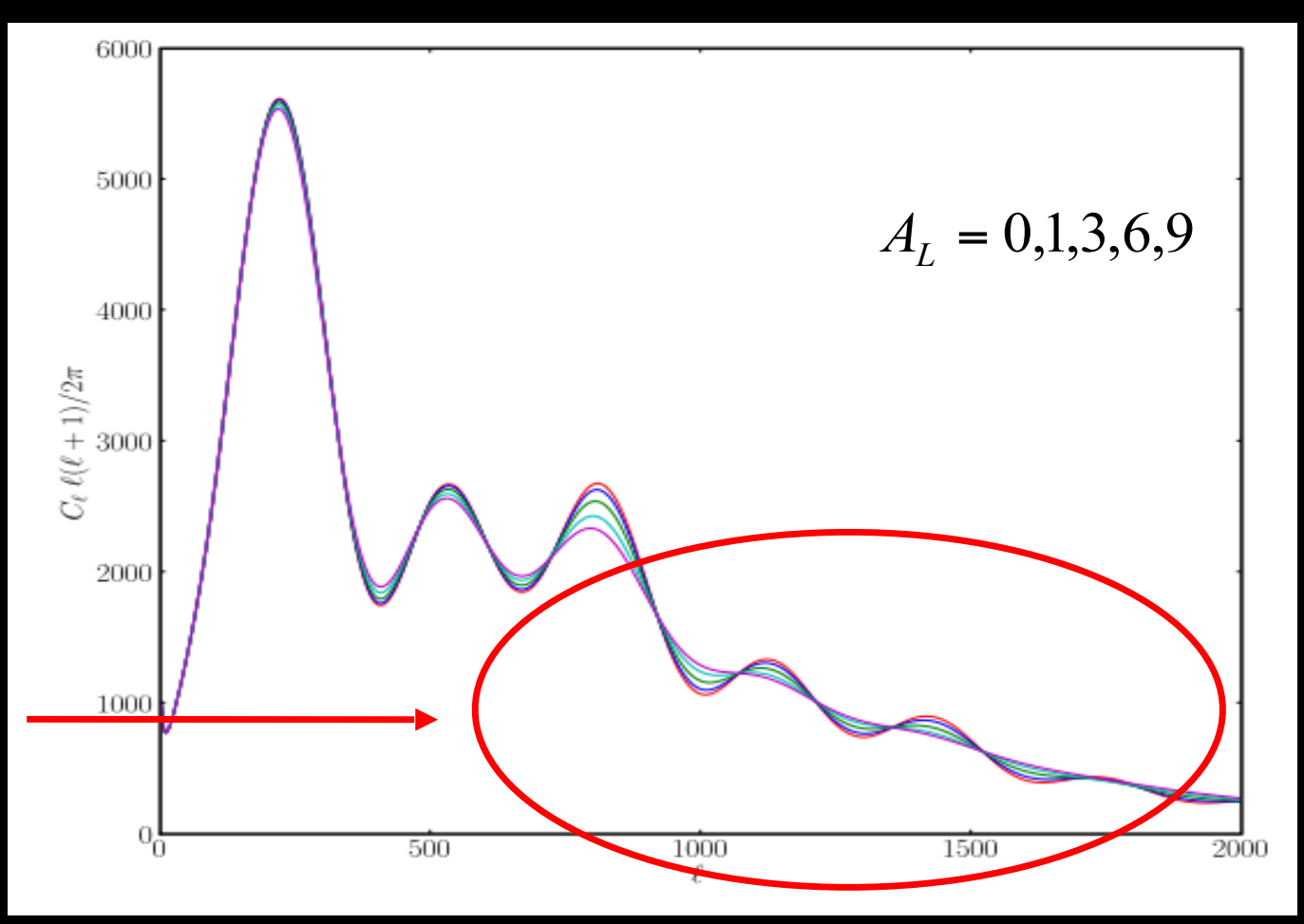

Calabrese et al., Phys. Rev. D, 77, 123531 


\section{$A_{L}$ internal anomaly}

The Planck lensing-reconstruction power spectrum is consistent with the amplitude expected for LCDM models that fit the CMB spectra, so the Planck lensing measurement is compatible with $A L=1$.

However, the distributions of AL inferred from the $\mathrm{CMB}$ power spectra alone indicate a preference for $A L>1$.

The joint combined likelihood shifts the value preferred by the TT data downwards towards $A L=1$, but the error also shrinks, increasing the significance of $A L>1$ to $2.8 \sigma$.

The preference for high $A L$ is not just a volume effect in the full parameter space, with the best fit improved by $\Delta \mathrm{X}^{2} \sim 9$ when adding $A L$ for TT+lowE and 10 for TTTEEE+lowE.

Planck 2018, Aghanim et al., arXiv:1807.06209 [astro-ph.CO]

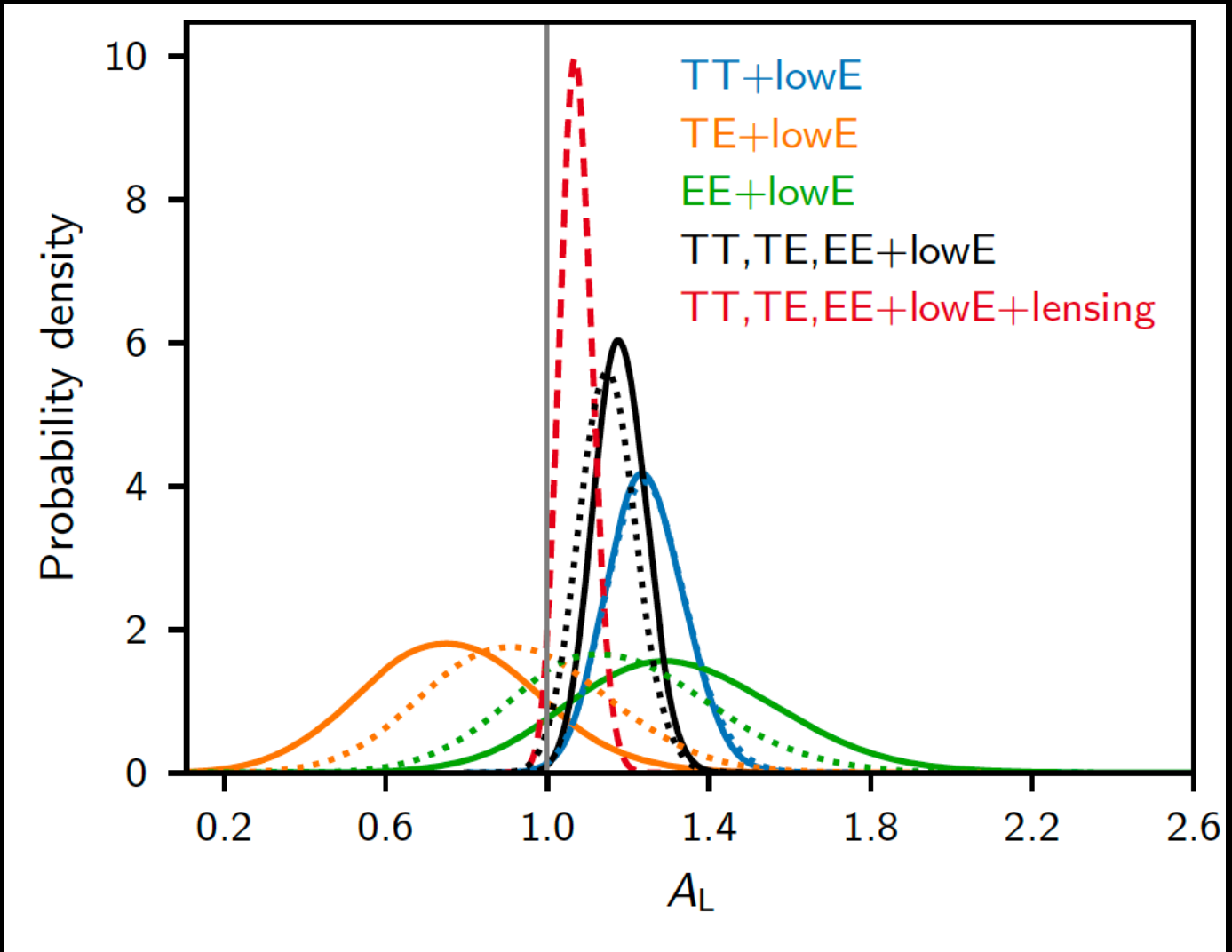

$$
\begin{aligned}
& A_{\mathrm{L}}=1.243 \pm 0.096 \quad(68 \%, \text { Planck TT+lowE }), \\
& A_{\mathrm{L}}=1.180 \pm 0.065 \quad(68 \%, \text { Planck TT,TE,EE+lowE }),
\end{aligned}
$$




\section{$A_{L}$ internal anomaly}
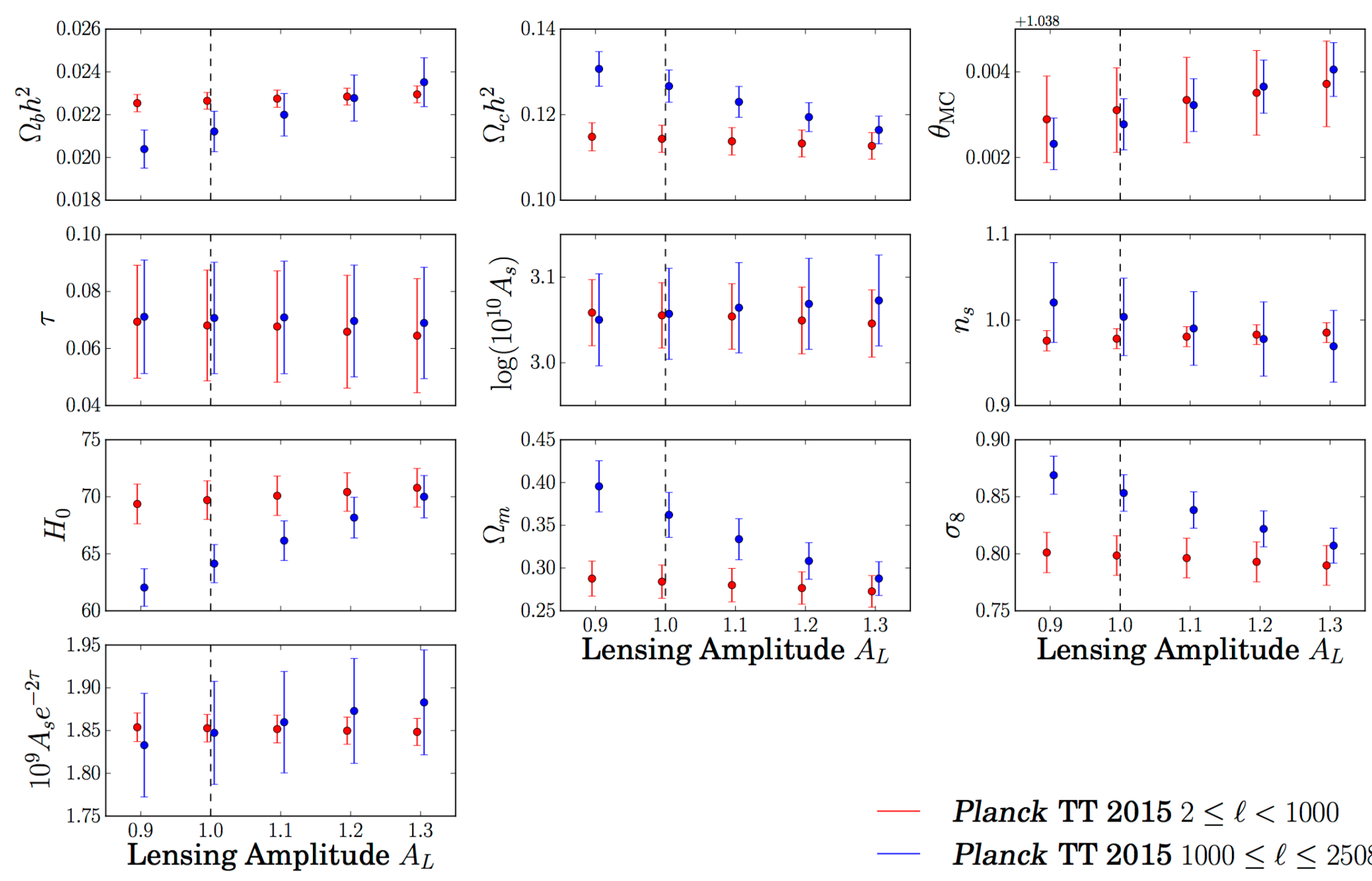

- Planck TT $20152 \leq \ell<1000$

— Planck TT $20151000 \leq \ell \leq 2508$

Marginalized $68.3 \%$ confidence $\Lambda$ CDM parameter constraints from fits to the $\mathrm{I}<1000$ and I $\geq 1000$ Planck TT 2015 spectra, fixing AL at different values. Tension at more than $2 \sigma$ level is apparent in $\Omega_{c} h^{2}$ and derived parameters, including $\mathrm{HO}, \Omega \mathrm{m}$, and $\sigma 8$. 


\section{$A_{L}$ internal anomaly}

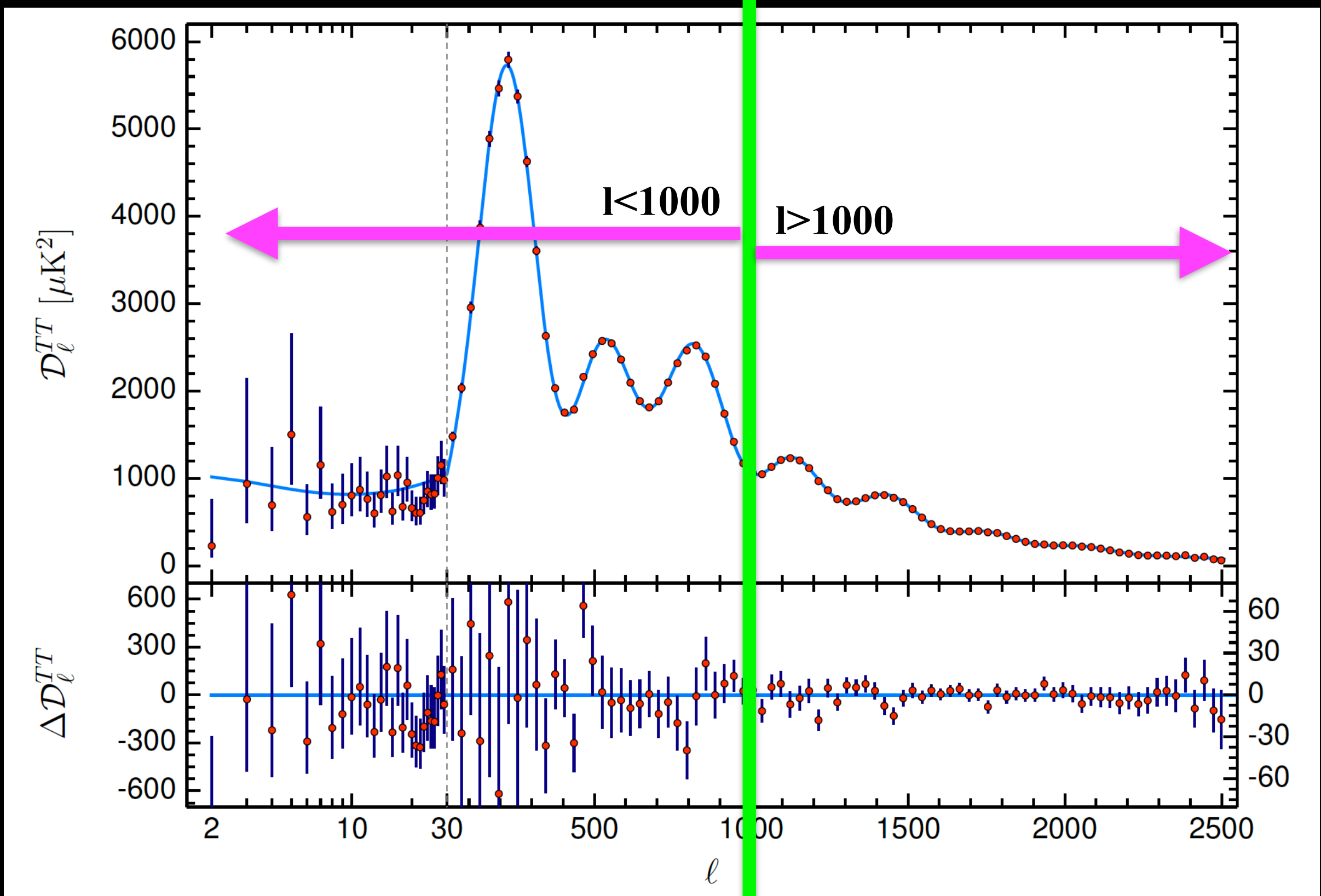




\section{$A_{L}$ internal anomaly}
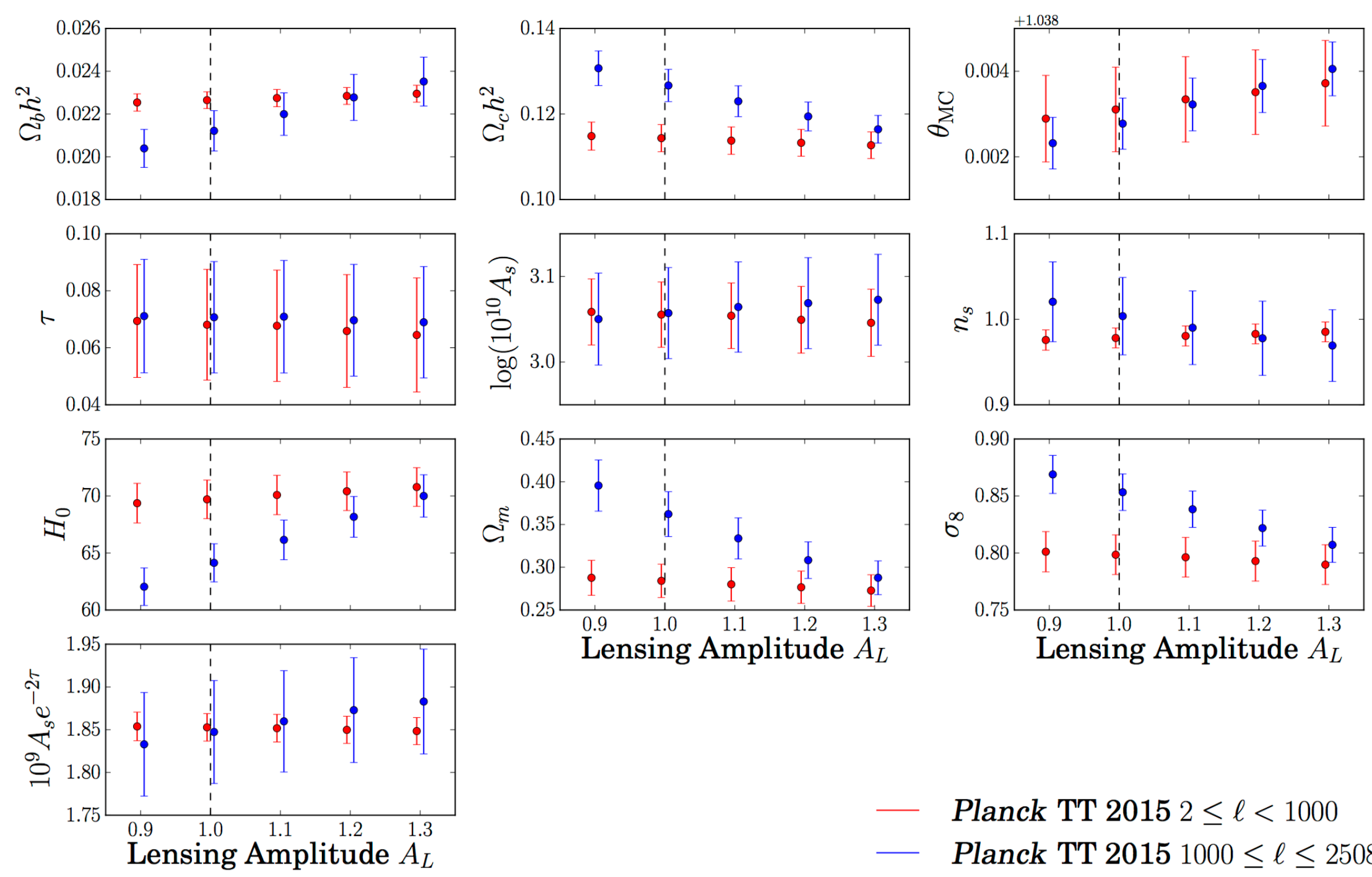

- Planck TT $20152 \leq \ell<1000$

- Planck TT $20151000 \leq \ell \leq 2508$

Increasing AL smooths out the high order acoustic peaks, improving the agreement between the two multipole ranges. 


\section{S8 tension}
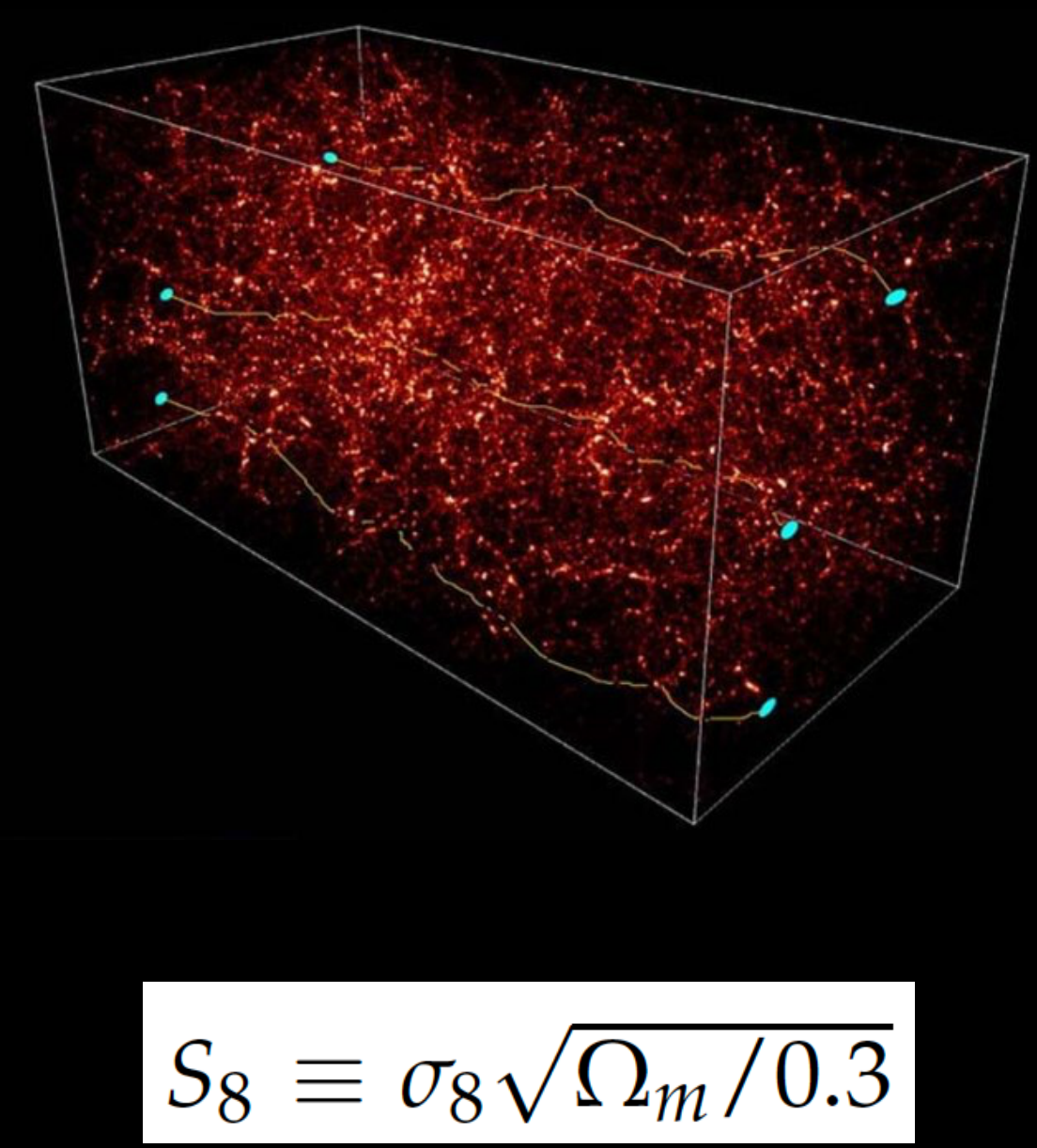

A tension on $\mathrm{S} 8$ is present between the Planck data in the $\Lambda$ CDM scenario and the cosmic shear data. 


\section{S8 tension}

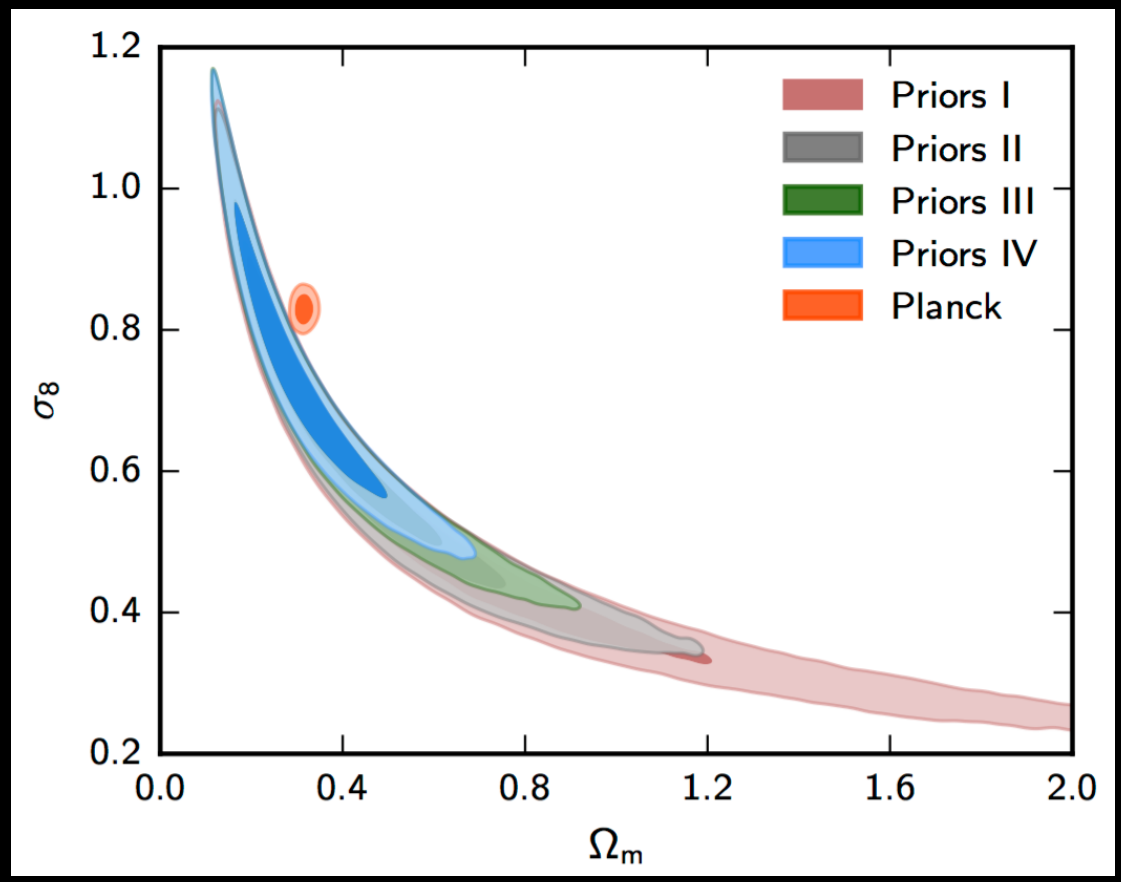

Joudaki et al, arXiv:1601.05786

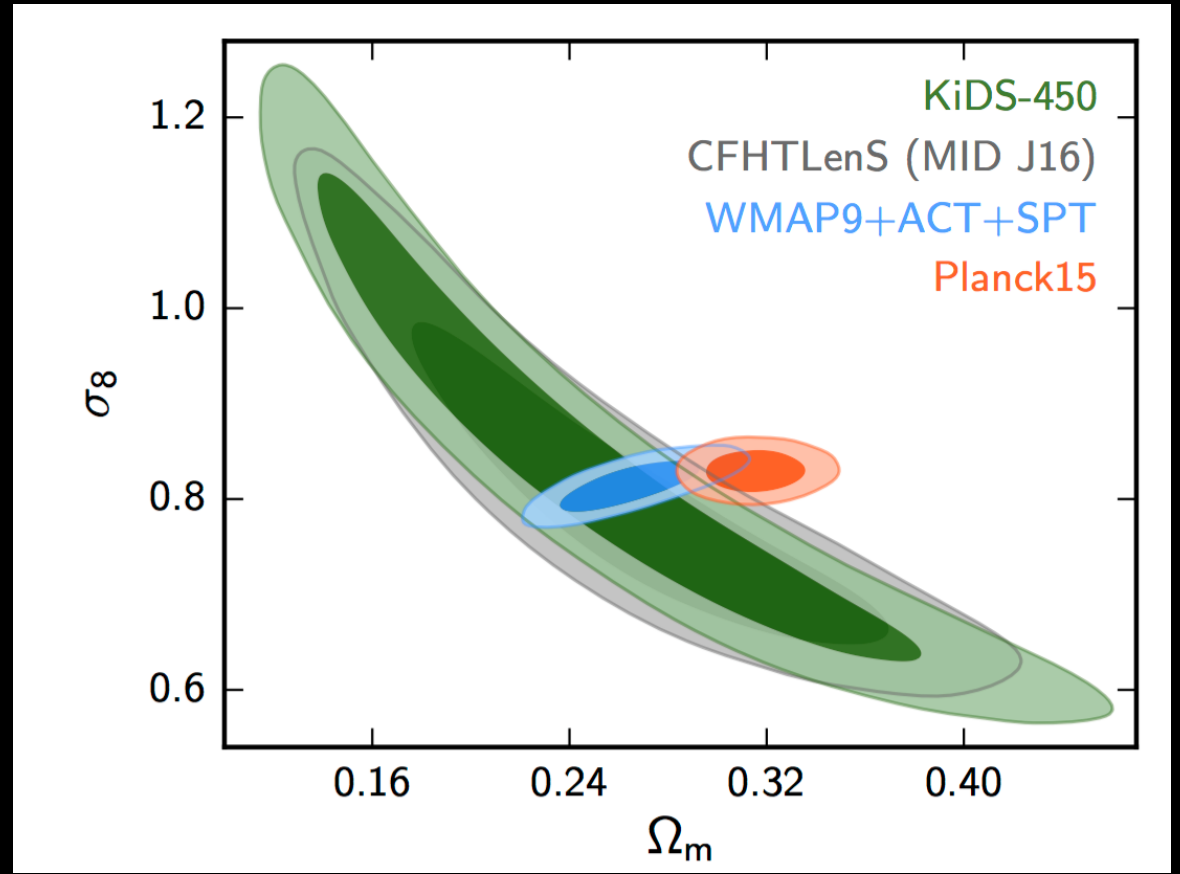

Hildebrandt et al., arXiv:1606.05338.

$$
S_{8} \equiv \sigma_{8} \sqrt{\Omega_{m} / 0.3}
$$

The S8 tension is at about 2.6 sigma level between the Planck data in the $\Lambda$ CDM scenario and CFHTLenS survey and KiDS-450. 


\section{The S8 tension}

This is mainly due to the anomalous value of $A_{L}$.

We find that the $\mathrm{CMB}$ and cosmic shear datasets, in tension in the standard LCDM model, are still in tension adding massive neutrinos.

However, if we include the additional scaling parameter on the CMB lensing amplitude $A_{L}$, we find that this can put in agreement the Planck 2015 with the cosmic shear data.

$A_{L}$ is a phenomenological parameter that is found to be more than $2 \sigma$ higher than the expected value in the Planck 2015 data, suggesting a higher amount of lensing in the power spectra, not supported by the trispectrum analysis.

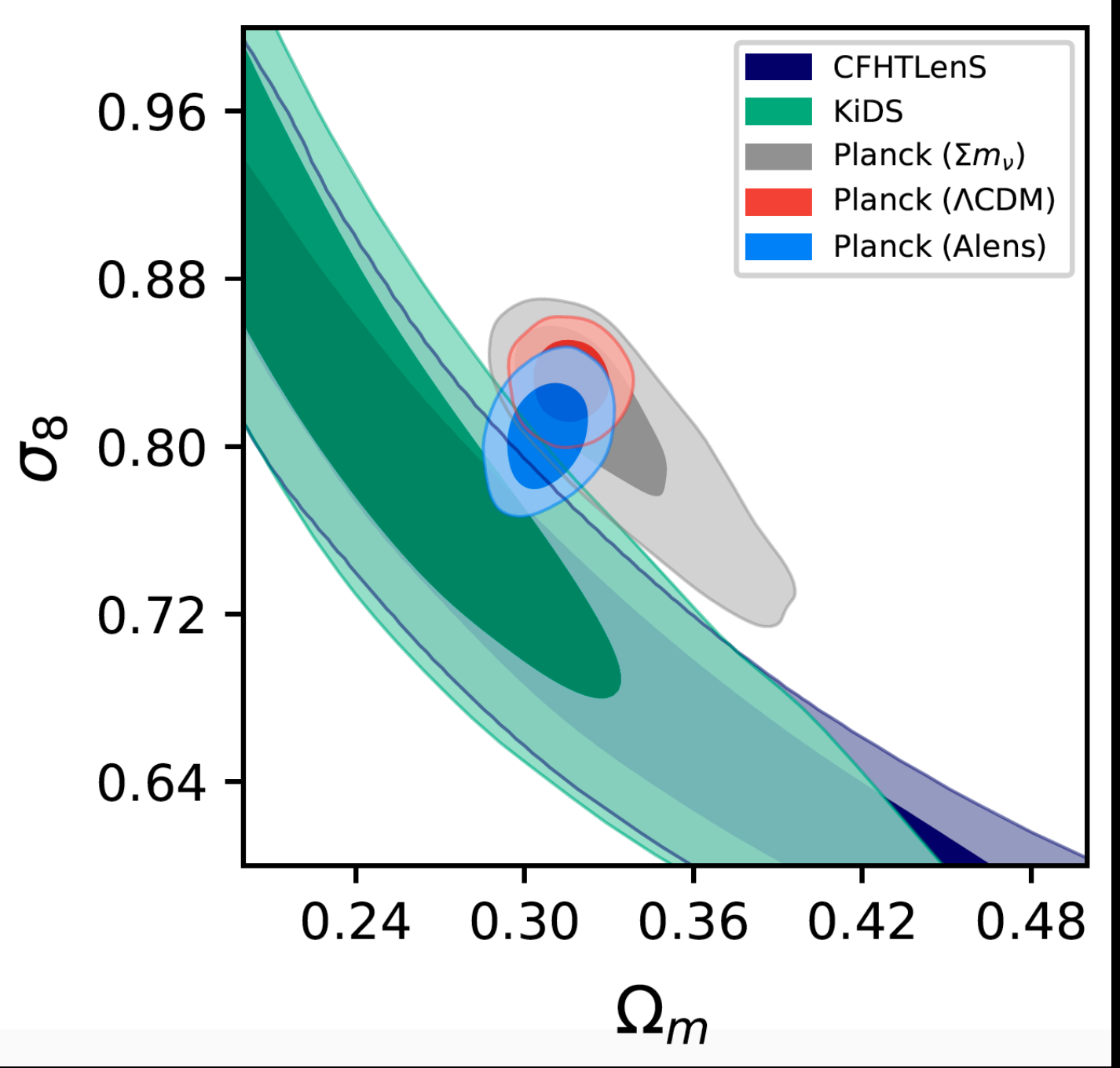

Di Valentino and Bridle, Symmetry 10 (2018) no.11, 585 


\section{The S8 tension}

This is mainly due to the anomalous value of $A_{L}$.

We find that the $\mathrm{CMB}$ and cosmic shear datasets, in tension in the standard LCDM model, are still in tension adding massive neutrinos.

However, if we include the additional scaling parameter on the CMB lensing amplitude $A_{L}$, we find that this can put in agreement the Planck 2015 with the cosmic shear data.

$A_{L}$ is a phenomenological parameter that is found to be more than $2 \sigma$ higher than the expected value in the Planck 2015 data, suggesting a higher amount of lensing in the power spectra, not supported by the trispectrum analysis.

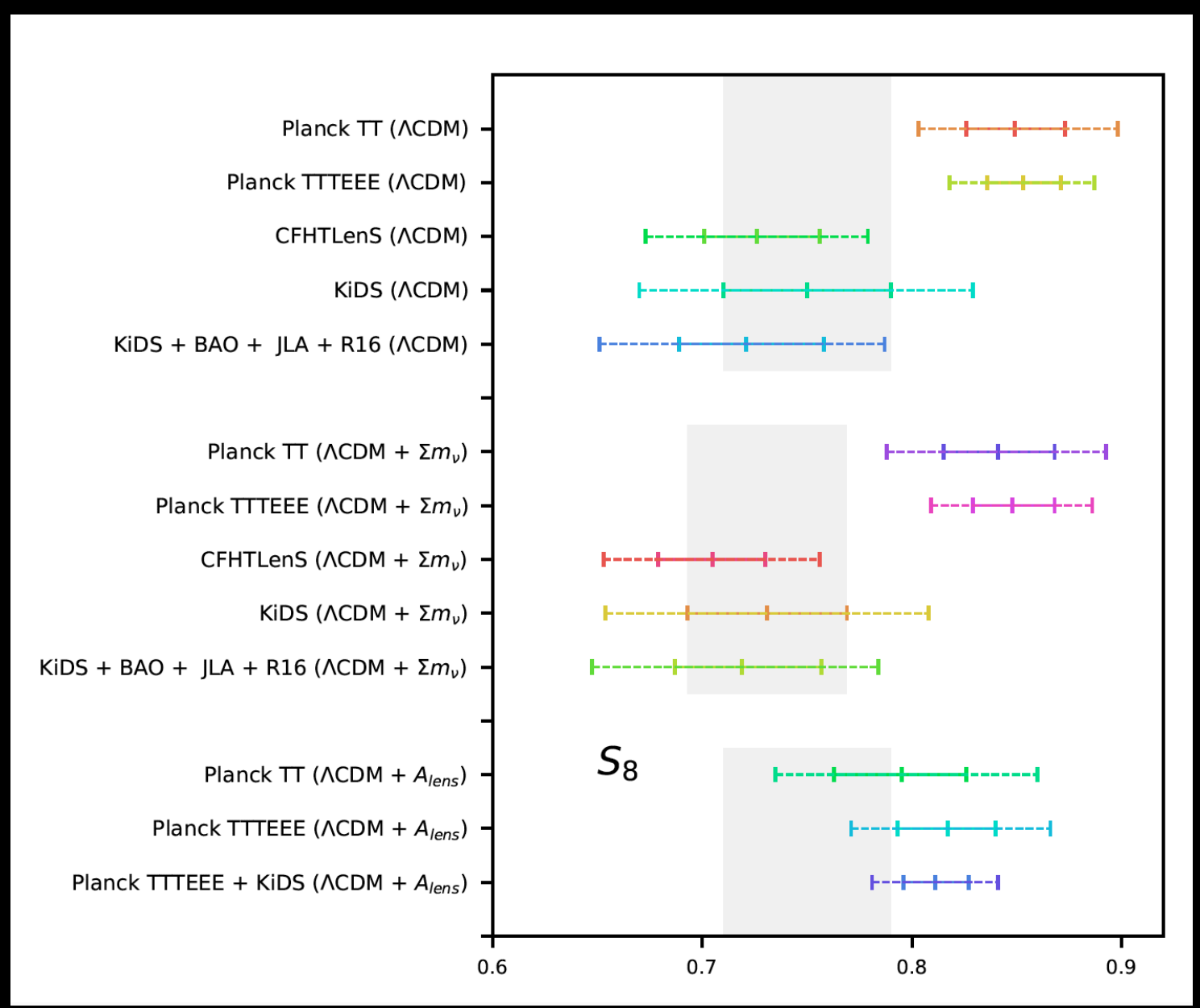

Di Valentino and Bridle, Symmetry 10 (2018) no.11, 585 


\section{Curvature of the universe}

The ACDM model assumes that the universe is specially flat. The combination of the

Planck temperature and polarization power spectra give

$$
\Omega_{K}=-0.044_{-0.015}^{+0.018} \quad(68 \%, \text { Planck TT,TE,EE+lowE }),
$$

a detection of curvature at about $3.4 \sigma$.

This is not entirely a volume effect, since the best-fit $\Delta \chi^{2}$ changes by -11 compared to base $\Lambda$ CDM when adding the one additional curvature parameter. 


\section{Curvature of the universe}

The reasons for the pull towards negative values of $\Omega_{k}$ are essentially the same as those that lead to the preference for $A L>1$, although slightly exacerbated in the case of curvature, since the low multipoles also fit the low-temperature likelihood slightly better if $\Omega_{K}<0$.

In fact, closed models predict substantially higher lensing amplitudes than in $\Lambda C D M$, because the dark matter content can be greater, leading to a larger lensing signal.

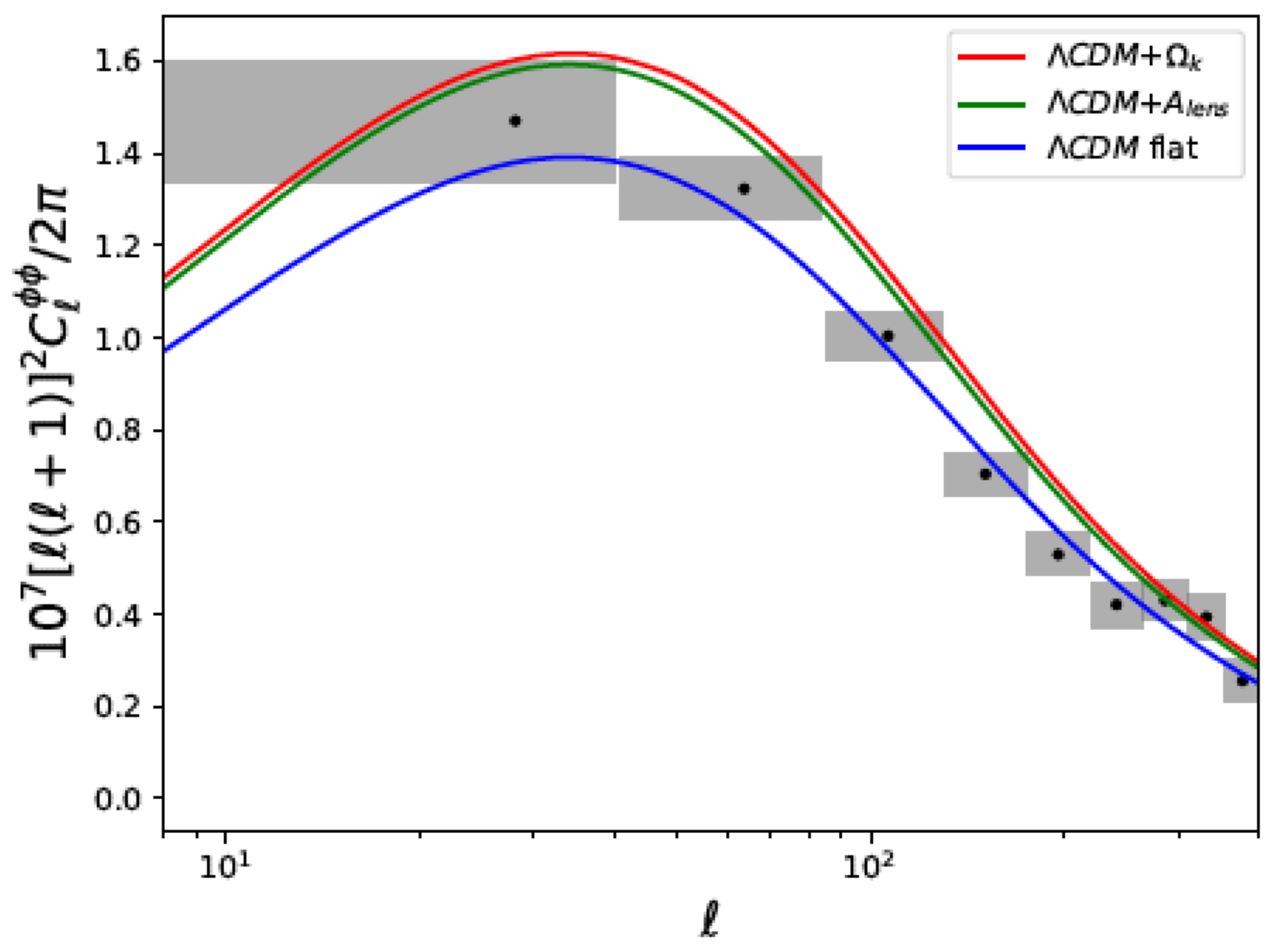

Di Valentino, Melchiorri and Silk, Nature Astronomy (2019) 


\section{Curvature of the universe}

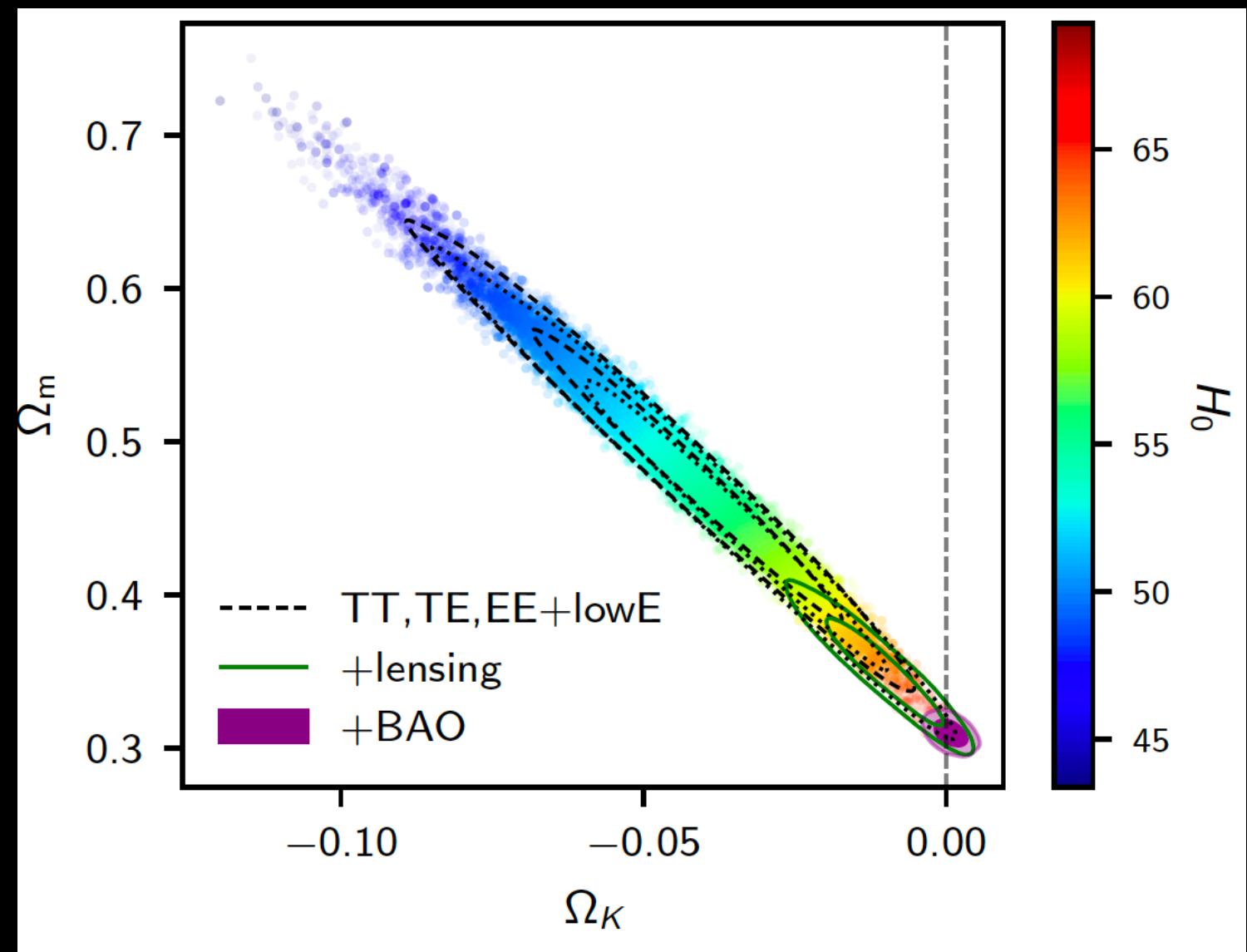

Adding BAO data, filled contours, convincingly breaks the geometric degeneracy giving a joint constraint very consistent with a flat universe.

$$
\begin{array}{ll}
\Omega_{K}=0.0007 \pm 0.0019 & \begin{array}{l}
(68 \%, \mathrm{TT}, \mathrm{TE}, \mathrm{EE}+\text { lowE } \\
+ \text { lensing }+\mathrm{BAO})
\end{array}
\end{array}
$$




\section{Curvature of the universe}

To compare the Planck results with other experiments we first check if Planck can provide an unbiased and reliable estimate of the curvature of the Universe. This may not be the case since a "geometrical degeneracy" is present with $\Omega m$.

When precise CMB measurements at arcminute angular scales are included, since gravitational lensing depends on the matter density, its detection breaks the geometrical degeneracy. The Planck experiment with its improved angular resolution offers the unique opportunity of a precise measurement of curvature from a single $\mathrm{CMB}$ experiment. We simulated Planck, finding that such experiment could constrain curvature with a $2 \%$ uncertainty, without any significant bias towards closed models.

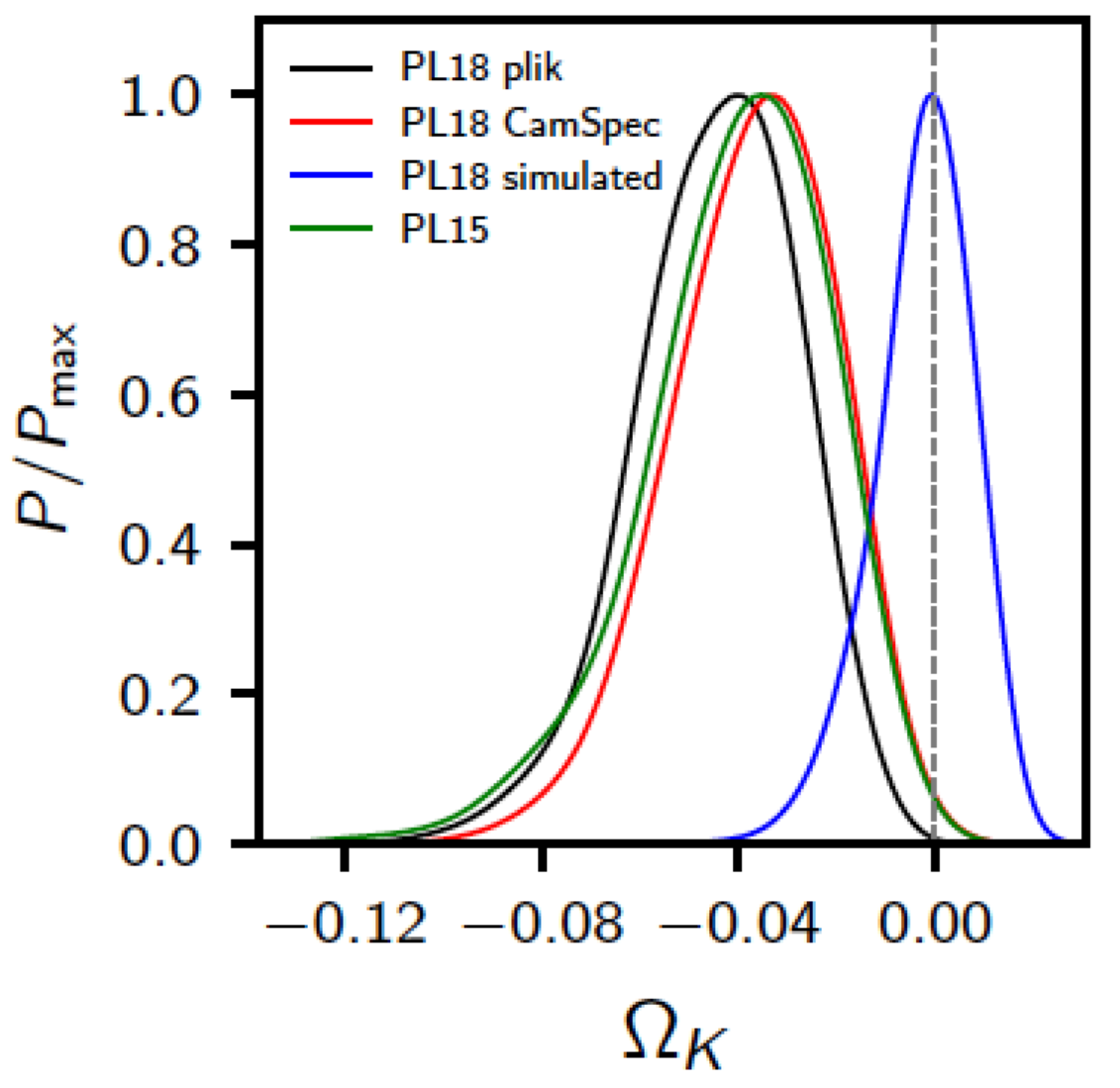

Di Valentino, Melchiorri and Silk, Nature Astronomy (2019) 


\section{Curvature of the universe}

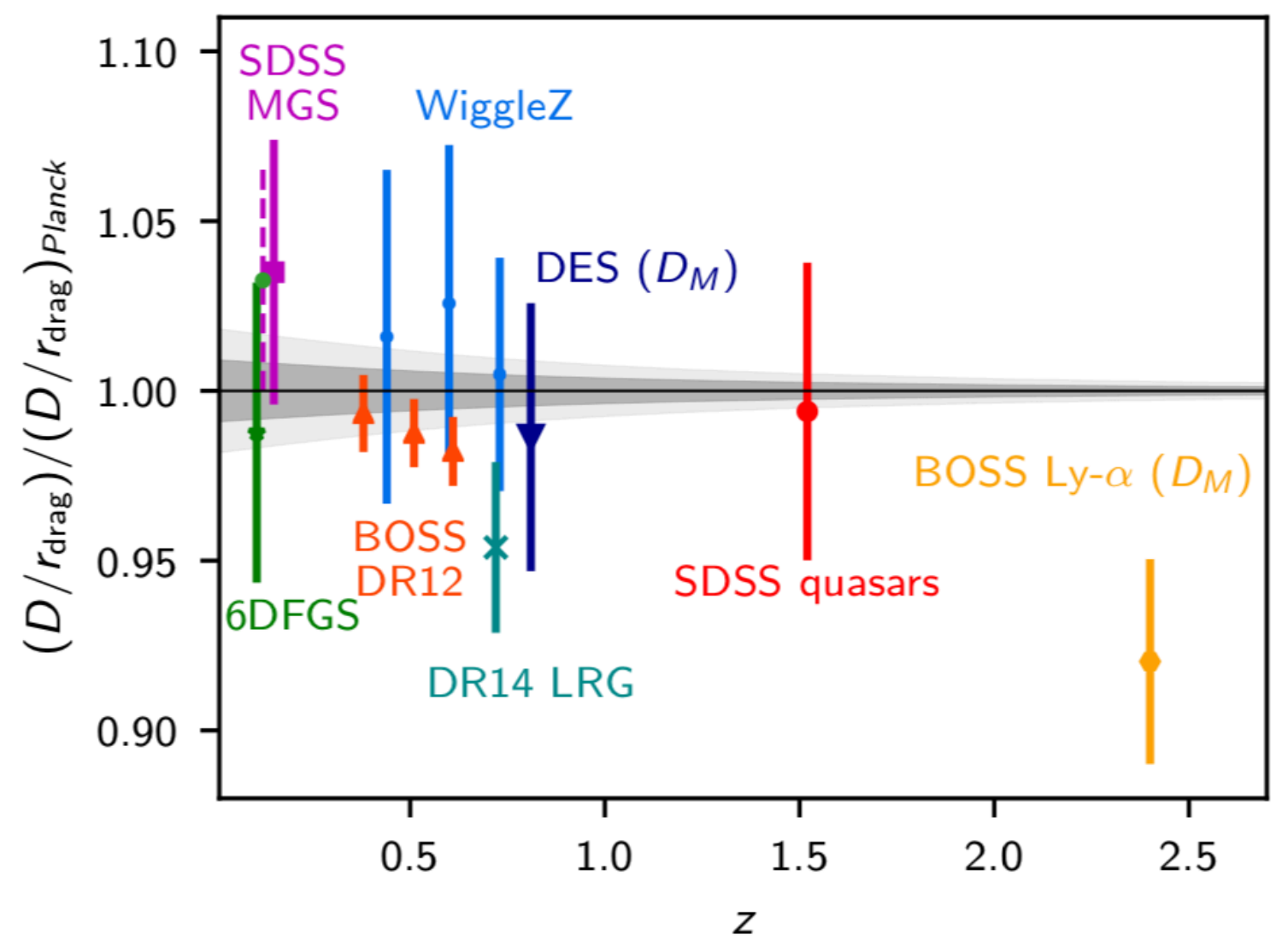

Planck 2018, Aghanim et al., arXiv:1807.06209 [astro-ph.CO]

In a $\Lambda$ CDM model the BAO data agree really well with the Planck measurements... 


\section{Curvature of the universe}

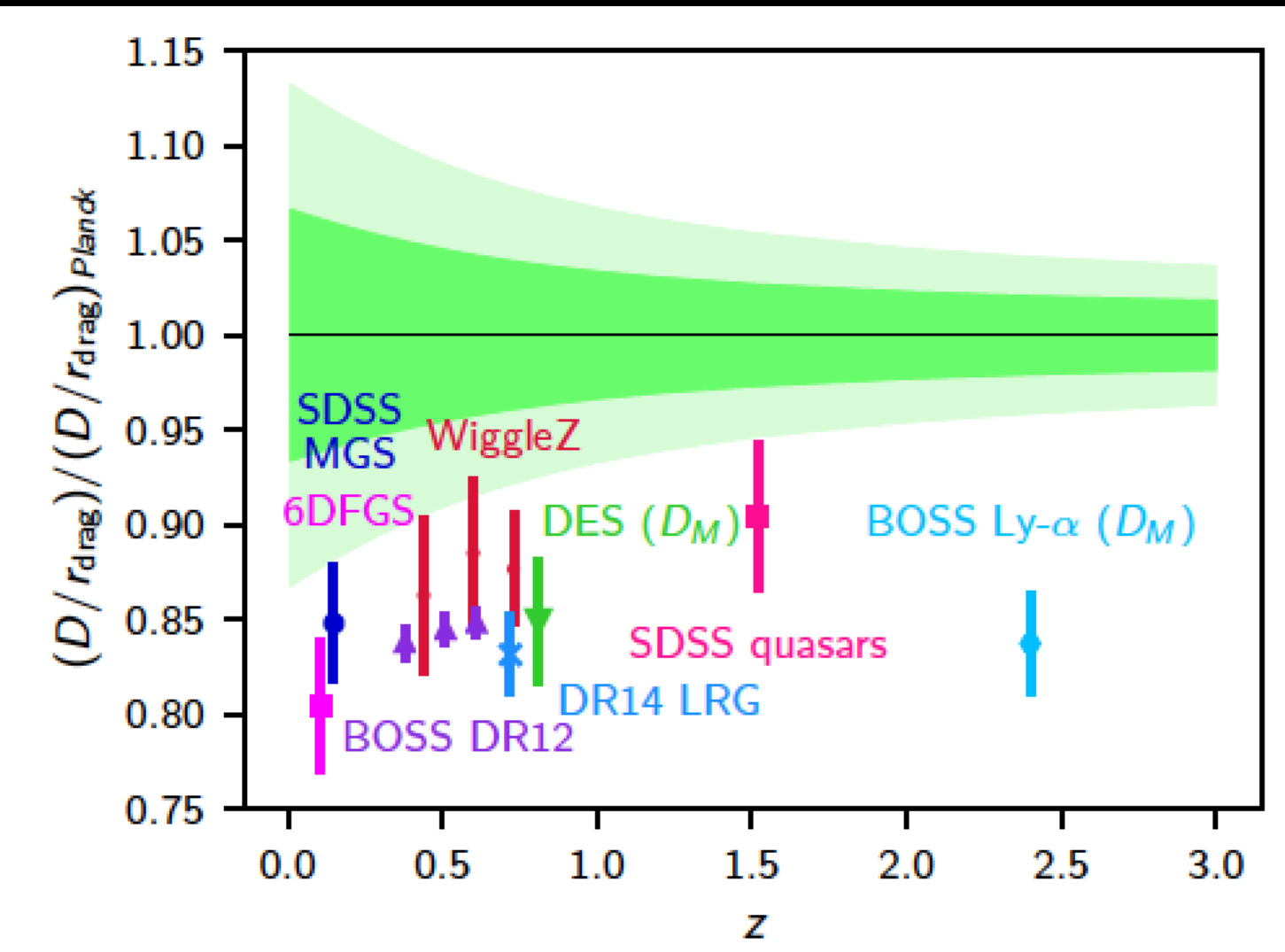

Di Valentino, Melchiorri and Silk, Nature Astronomy (2019)

when we let curvature to vary...

Planck spectra are inconsistent with BAO measurements at more than 30 ! The assumption of a flat universe could therefore mask a cosmological crisis where disparate observed properties of the Universe appear to be mutually inconsistent. 


\section{Curvature can explain $A_{L}$}

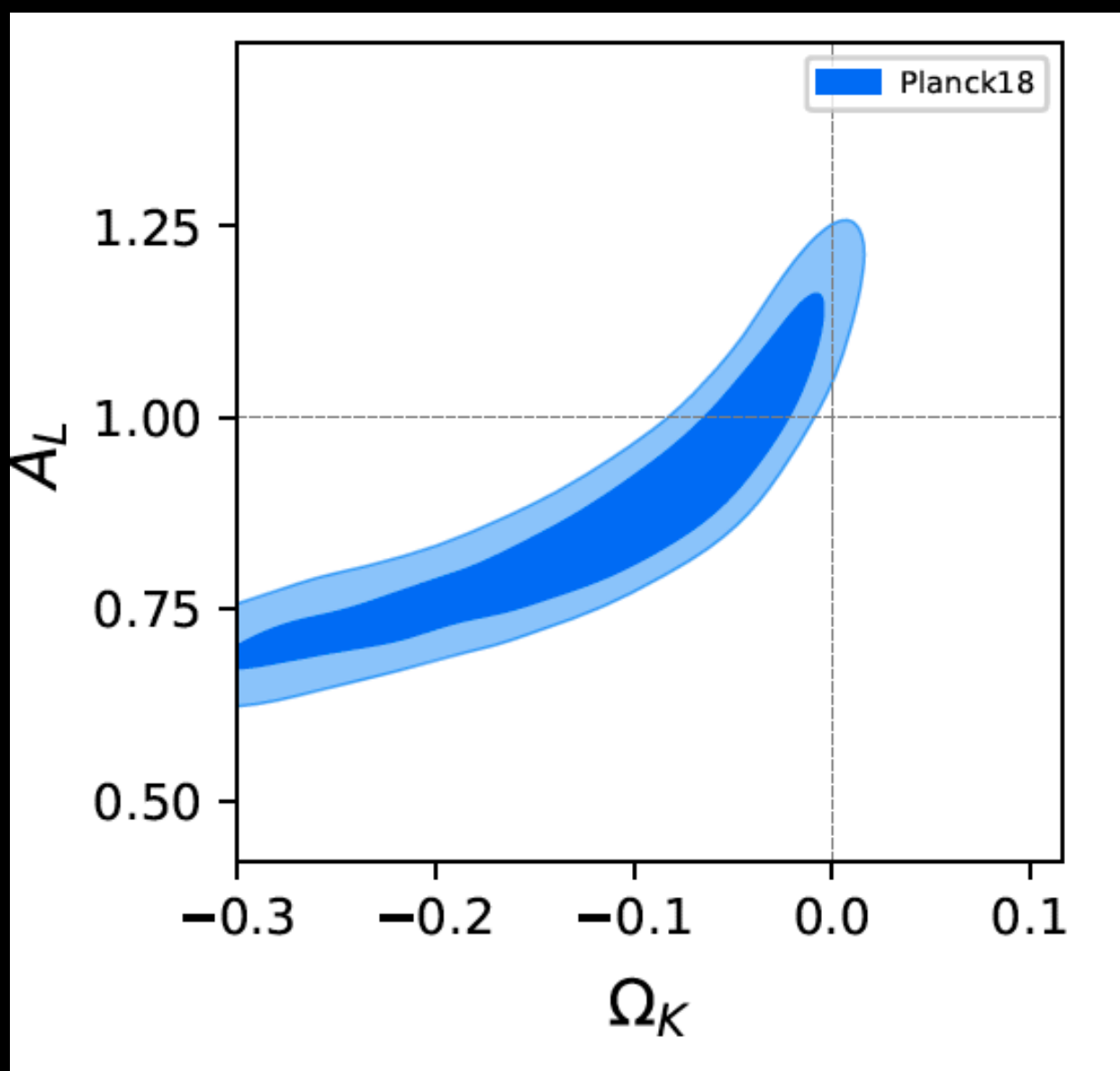

Di Valentino, Melchiorri and Silk, Nature Astronomy (2019)

A degeneracy between curvature and the AL parameter is clearly present. A closed universe can provide a physical explanation to the enhancement of the lensing amplitude. Note that a model with $\Omega K<0$ is slightly preferred with respect to a flat model with $\mathrm{AL}>1$. 


\section{Curvature can explain internal tension}

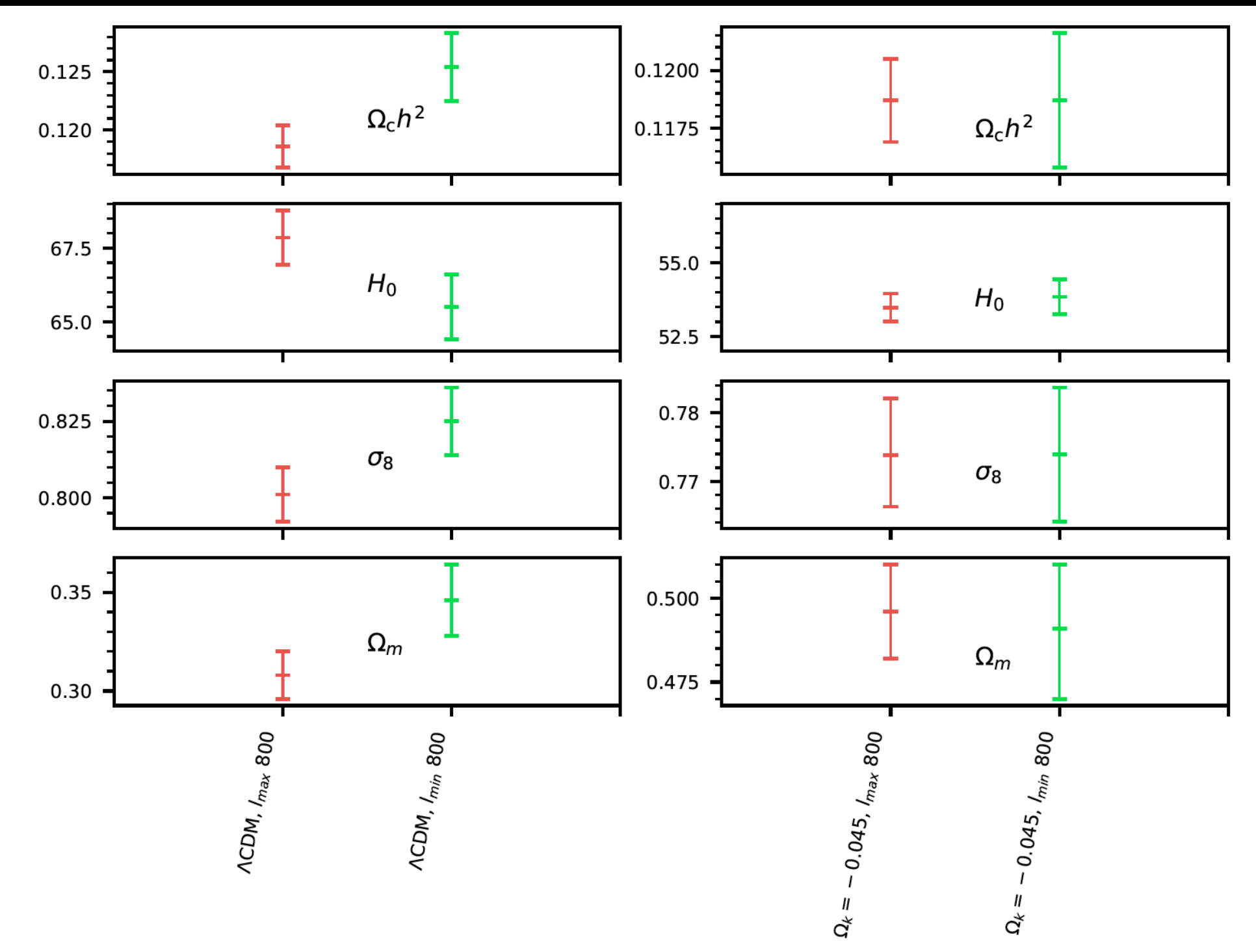

Di Valentino, Melchiorri and Silk, Nature Astronomy (2019)

Fixing $\Omega K$ to the best fit value we improve the agreement between the two multipole ranges. 


\section{Curvature can't explain external tensions}
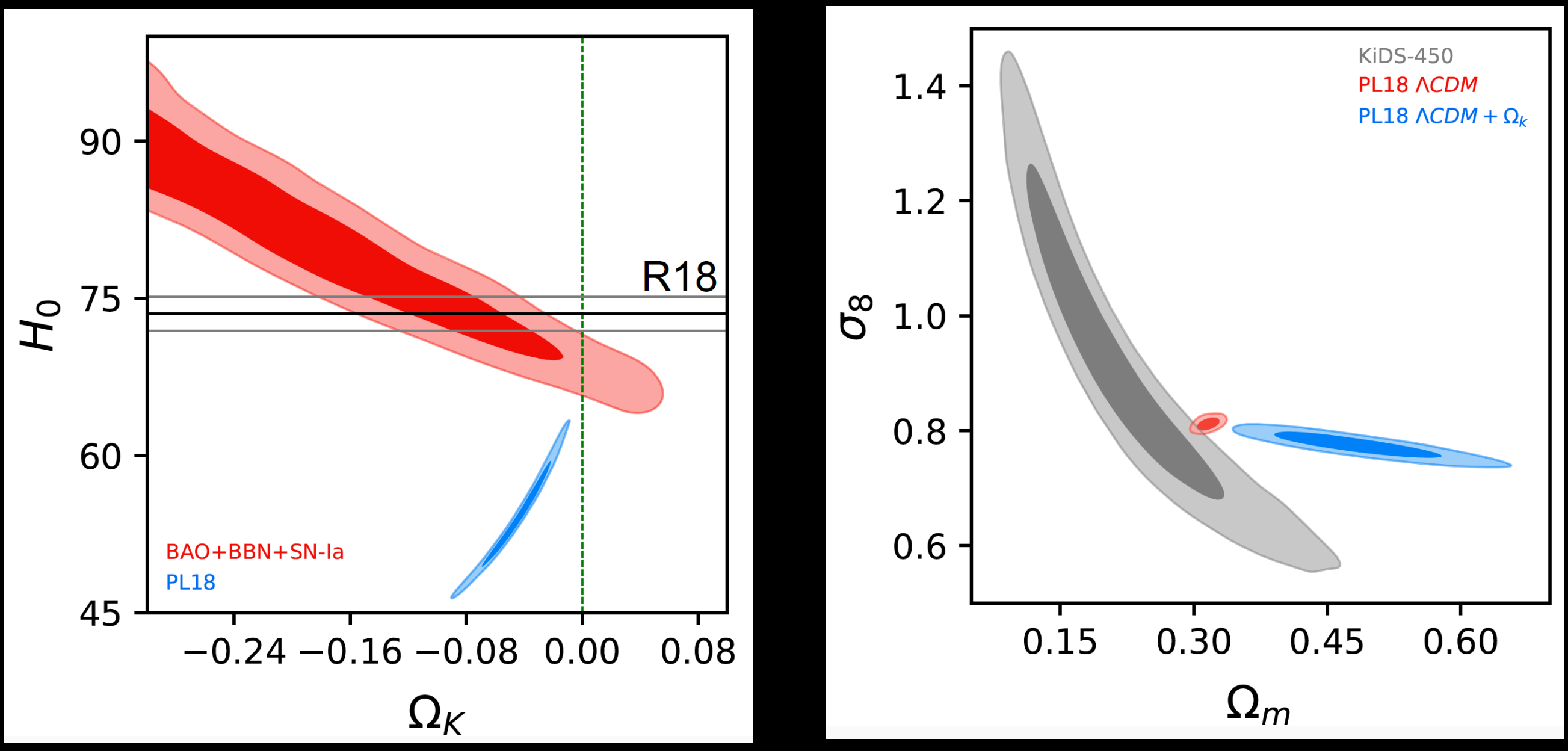

Di Valentino, Melchiorri and Silk, Nature Astronomy (2019)

However, varying $\Omega k$ the well know tensions on $\mathrm{H0}$ and $\mathrm{S} 8$ are exacerbates. 


\section{Summarising}

Extended neutrino scenarios seem no more suitable for solving the $\mathrm{HO}$ tension when the Planck polarisation is considered, but a phantom like dark energy equation of state is still OK.

We studied a simple IDE model that relieve the $\mathrm{HO}$ tension hinting for an interaction different from zero at more than $5 \sigma$. Even when BAO data are added in the analysis the Hubble constant tension is reduced at less than $3 \sigma$.

The excess of lensing in the Planck temperature power spectrum seems to be responsible for the tension with the cosmic shear data.

An indication at more than 3 standard deviation is present in the Planck $2018 \mathrm{CMB}$ data for a closed universe than can explain the excess of lensing, but increases the tension with the other cosmological probes.

In order to have a new concordance model, next decade of experiments will be decisive. 


\section{For any questions:}

eleonora.divalentino@manchester.ac.uk 


\section{References}

- Aghanim et al. [Planck Collaboration], arXiv:180\%.06209 [astro-ph.CO];

- Bennett et al. [WMAP collaboration], arXiv:1212.5225 [astro-ph.CO];

- Ade et al. [Planck Collaboration], arXiv:1502.01598 [astro-ph.CO];

- Aghanim et al. [Planck Collaboration], arXiv:1605.02985 [astro-ph.CO];

- Riess et al. arXiv:1604.01424v3;

- Riess et al. arXiv:1801.01120 [astro-ph.SR];

- Riess et al. 2018, ApJ, 861, 126

- Wong et al. arXiv:190\%.04869v1

- Riess et al. arXiv:1903.07603 [astro-ph.CO];

- Dhawan et al. Astron.Astrophys. 609 (2018) A72

- Burns et al. [CSP collaboration], Astrophys.J. 869 (2018) no.1, 56

- Joudaki et al, arXiv:1601.05786;

- Hildebrandt et al., arXiv:1606.05338 [astro-ph.CO];

- Mangano et al., Nucl. Phys. B 729, 221 (2005) [hep$\mathrm{ph} / 0506164]$;

- Archidiacono et al. Adv.High Energy Phys. 2013 (2013) 19104\%;

- Calabrese et al., Phys. Rev. D, r77, 123531;

- Di Valentino and Bridle, Symmetry 10 (2018) no.11, 585

- Di Valentino et al., Phys.Rev. D96 (2017) no.2, 023523;
- Heymans et al., Mon. Not. Roy. Astron. Soc. 427, 146 (2012);

- Erben et al., Mon. Not. Roy. Astron. Soc. 433, 2545 (2013);

- Beutler et al., Mon. Not. Roy. Astron. Soc. 416, 3017 (2011);

- Ross et al., Mon. Not. Roy. Astron. Soc. 449, no. 1, 835 (2015);

- Anderson et al. [BOSS Collaboration], Mon. Not. Roy. Astron. Soc. 441, no. 1, 24 (2014);

- M. Betoule et al. [SDSS Collaboration] Astron. Astrophys 568, A22 (2014);

- Gavela et al. J. Cosmol. Astropart. Phys. Ory (2009) 034

- Di Valentino et al. Phys.Rev. D96 (2017) no.4, 043503

- Abbott et al. arXiv:1710.05835 [astro-ph.CO]

- Adam et al. [Planck Collaboration], arXiv:1502.01582 [astro-ph.CO];

- Aghanim et al. [Planck Collaboration], arXiv:150\%.02704 [astro-ph.CO];

- Aghanim et al. [Planck Collaboration], arXiv:1605.02985 [astro-ph.CO];

- Di Valentino et al. arXiv:1908.04281

- Di Valentino et al. arXiv:1910.09853

- Di Valentino, Melchiorri and Silk, Nature Astronomy 2019. 